\title{
Practical Examples for Improved Transmission in Helical Waveguides, Based on One Bending and Two Bendings
}

\author{
Z. Menachem ${ }^{1} \&$ S. Tapuchi ${ }^{1}$ \\ ${ }^{1}$ Department of Electrical Engineering, Sami Shamoon College of Engineering, Israel \\ Correspondence: Z. Menachem, Department of Electrical Engineering, Sami Shamoon College of Engineering, \\ Israel. E-mail: zionm@post.tau.ac.il
}

Received: December 26, 2011 Accepted: February 2, 2012 Online Published: May 1, 2012

doi:10.5539/apr.v4n2p233 URL: http://dx.doi.org/10.5539/apr.v4n2p233

\begin{abstract}
This paper presents an improved approach for the propagation of electromagnetic (EM) fields along a helical hollow waveguide, based on one bending and two bendings. The objective is to develop a mode model for infrared (IR) wave propagation, in order to represent the effect of the radius of the cylinder of the helix and the step's angle on the output fields and the output power transmission. This model enables us to understand more precisely the influence of the step's angle and the radius of the cylinder of the helix on the output results of each section of the helical waveguide, based on one bending and two bendings, for practical cases. The output power transmission for all bending is improved by increasing the step's angle or the radius of the cylinder of the helix, especially in the cases of space curved waveguides. This mode model can be a useful tool to improve the output results in all the cases of the helical hollow waveguides, based on one bending and two bendings, for industrial and medical regimes.
\end{abstract}

Keywords: wave propagation, helical waveguide, dielectric waveguide, power transmission

\section{Introduction}

Cylindrical hollow metallic and metallic with inner dielectric coating waveguide have been proposed in the literature. A review of the hollow waveguide technology (Harrington \& Matsuura Y., 1995) (Harrington, Harris, \& Katzir, 1995) and a review of IR transmitting, hollow waveguides, fibers and integrated optics (Harrington, 2000) were published. The first theoretical analysis of the problem of hollow cylindrical bent waveguides was published by Marcatili and Schmeltzer (Marcatili \& Schmeltzer, 1964), where the theory considers the bending as a small disturbance and uses cylindrical coordinates to solve Maxwell equations. They derive the mode equations of the disturbed waveguide using the ratio of the inner radius $\mathrm{r}$ to the curvature radius $\mathrm{R}$ as a small parameter $(\mathrm{r} / \mathrm{R} \ll$ 1). Their theory predicts that the bending has little influence on the attenuation of a hollow metallic waveguide. Marhic (1981) proposed a mode-coupling analysis of the bending losses of circular metallic waveguide in the IR range for large bending radii. In the circular guide it is found that the preferred $T E_{01}$ mode can couple very effectively to the lossier $T M_{11}$ mode when the guide undergoes a circular bend. For circular waveguides, the microwave approximation has been used for the index of refraction and the straight guide losses, and the results indicate very poor bending properties due to the near degeneracy of the $T E_{01}$ and $T M_{11}$ modes, thereby offering an explanation for the high losses observed in practice.

Miyagi et al. (1984) suggested an improved solution, which provided agreement with the experimental results, but only for $\mathrm{r} / \mathrm{R} \ll 1$. A different approach (Marhic, 1981) (Croitoru, 1986) treats the bending as a perturbation that couples the modes of a straight waveguide. That theory explains the large difference between the metallic and metallic-dielectric bent waveguide attenuation. The reason for this difference is that in metallic waveguides the coupling between the TE and TM modes caused by the bending mixes modes with very low attenuation and modes with very high attenuation, whereas in metallic-dielectric waveguides, both the TE and TM modes have low attenuation.

Hollow waveguides with both metallic and dielectric internal layers were proposed to reduce the transmission losses. Hollow-core waveguides have two possibilities. The inner core materials have refractive indices greater than one (namely, leaky waveguides) or the inner wall material has a refractive index of less than one. A hollow waveguide can be made, in principle, from any flexible or rigid tube (plastic, glass, metal, etc.) if its inner hollow 
surface (the core) is covered by a metallic layer and a dielectric overlayer. This layer structure enables us to transmit both the TE and TM polarization with low attenuation (Marhic, 1981) (Croitoru, 1986).

A method for the EM analysis of bent waveguides (Melloni et al., 2001) is based on the expansion of the bend mode in modes of the straight waveguides, including the modes under the cutoff. A different approach to calculate the bending losses in curved dielectric waveguides (Bienstman et al., 2002) is based on the well-known conformal transformation of the index profile and on vectorial eigenmode expansion combined with perfectly matched layer boundary conditions to accurately model radiation losses. An improved ray model for simulating the transmission of laser radiation through a metallic or metallic dielectric multibent hollow cylindrical waveguide was proposed (Mendlovic et al., 1989) (Morhaim et al., 1991). It was shown theoretically and proved experimentally that the transmission of $\mathrm{CO}_{2}$ laser radiation is possible even through bent waveguide.

The propagation of EM waves in a loss-free inhomogeneous hollow conducting waveguide with a circular cross section and uniform plane curvature of the longitudinal axis was considered (Kark, 1991). For small curvature the field equations can be solved by means of an analytical approximation method. In this approximation the curvature of the axis of the waveguide was considered as a disturbance of the straight circular cylinder, and the perturbed torus field was expanded in eigenfunctions of the unperturbed problem. An extensive survey of the related literature can be found especially in the book on EM waves and curved structures (Lewin et al., 1977). The radiation from curved open structures is mainly considered by using a perturbation approach, that is by treating the curvature as a small perturbation of the straight configuration. The perturbative approach is not entirely suitable for the analysis of relatively sharp bends, such as those required in integrated optics and especially short millimeter waves.

The models based on the perturbation theory consider the bending as a perturbation $(\mathrm{r} / \mathrm{R} \ll 1)$, and solve problems only for a large radius of curvature.

Several methods of propagation along the toroidal and helical waveguides were developed, based on Maxwell's equations. The method of propagation along the toroidal waveguide (Menachem, 2003) has been derived for the analysis of EM wave propagation with arbitrary profiles, and with rectangular metal tubes. An improved approach has been derived for the propagation of EM field along a toroidal dielectric waveguide with a circular cross-section (Menachem et al., 2002). The method for the propagation of EM field along a helical dielectric waveguide with a circular cross section has been derived (Menachem \& Mond, 2006). The method for the propagation of EM field along a helical dielectric waveguide with a rectangular cross section has been derived (Menachem \& Haridim, 2009). It is very interesting to compare between the mode model methods for wave propagation in the waveguide with a rectangular cross section and with a circular cross section. The methods for one bending of the toroidal waveguide (approximately a plane curve) have been derived (Menachem, 2003) (Menachem et al., 2002) in the case of small values of step angle of the helix. The methods for one bending of the helical waveguide (a space curved waveguide) have been derived (Menachem \& Mond, 2006) (Menachem \& Haridim, 2009) for an arbitrary value of the step's angle of the helix. The method for the propagation of EM field along the toroidal waveguide that consists of two bendings in the same direction has been derived (Menachem, 2010) and the main steps of the method for the two bendings were introduced, in detail, for small values of step angles. The method of EM field along a helical hollow waveguide that consists of two bendings in the same direction has been derived (Menachem \& Tapuchi, 2010), and the main steps of this method were introduced, in detail, for an arbitrary value of the step's angle of the helix. The main point of the method (Menachem \& Tapuchi, 2010) was to generalize the method (Menachem, 2010) from a toroidal dielectric waveguide (approximately a plane curve) with two bendings to a helical waveguide (a space curved waveguide for an arbitrary value of the step's angle of the helix) with two bendings. The two above methods employ toroidal or helical coordinates (and not cylindrical coordinates, such as in the methods that considered the bending as a perturbation $(\mathrm{r} / \mathrm{R} \ll 1)$ ), and the calculations are based on using Laplace and Fourier transforms, and the output fields are computed by the inverse Laplace and Fourier transforms. Laplace transform on the differential wave equations is needed to obtain the wave equations (and thus also the output fields) that are expressed directly as functions of the transmitted fields at the entrance of the waveguide at $\zeta=0^{+}$. Thus, the Laplace transform is necessary to obtain the comfortable and simple input-output connections of the fields.

The main objective of this paper is to presents an improved approach for the propagation of EM fields along a helical hollow waveguide, based on one bending and two bendings. The main steps of the method for the helical waveguide with more than one bending (e.g., for two bendings) will be introduced in the derivation, for an arbitrary values of the step's angle (and not for small values of step angles). Our method employs helical coordinates 
(and not cylindrical coordinates, such as in the methods that considered the bending as a perturbation $(\mathrm{r} / \mathrm{R} \ll$ 1)). The derivation for all section of the helical waveguide is based on Maxwell's equations. The separation of variables is obtained by using the orthogonal-relations. The longitudinal components of the fields are developed into the Fourier-Bessel series. The transverse components of the fields are expressed as functions of the longitudinal components in the Laplace plane and are obtained by using the inverse Laplace transform by the residue method. In this paper, we supposed that the modes excited at the input of the waveguide by the conventional $\mathrm{CO}_{2}$ laser IR radiation $(\lambda=10.6 \mu \mathrm{m})$ are closer to the $T E M$ polarization of the laser radiation. The $T E M_{00}$ mode is the fundamental and the most important mode. This means that a cross-section of the beam has a Gaussian intensity distribution. The results of this model are applied to the study of helical hollow waveguides, based on one bending and two bendings, that are suitable for transmitting IR radiation, especially $\mathrm{CO}_{2}$ laser radiation.

\section{The Derivation}

This paper presents an improved approach for the propagation of EM fields along helical hollow waveguide, based on one bending and two bendings. Note that a helical waveguide relates to a space curved waveguide for an arbitrary value of the step's angle of the helix. Namely, the calculations in our method are dependent also on the arbitrary value of the step's angle of the helix. This model enables us to understand more precisely the influence of the step's angle and the radius of the cylinder of the helix on the output results of each section of the helical waveguide, based on one bending and two bendings.

Let us assume that the helical waveguide consists of two bendings in the same direction as shown in Figure 1(a). The helical circular waveguide is shown in Figure 1(b). The relevant main steps for calculations of the output fields and the output power transmission in the case of the helical waveguide with two bendings in the same direction are introduced in brief in this derivation, based on the method presented by Menachem and Tapuchi (Menachem \& Tapuchi, 2010). The coordinates of an arbitrary point on the toroidal system $(\mathrm{r}, \theta, \zeta)$ with a given bending (R) are shown in Figure 1(c), where $X=R \cos \phi$ and $Y=R \sin \phi$. The cross-section of the helical waveguide is shown in Figure 1(d).

Further we assume that the first bending (Figure 1(a)) is $R_{1}$, the length is $\zeta_{1}=\left(R_{1} \phi_{1}\right) / \cos \delta_{p}$, and the metric coefficient is $h_{\zeta_{1}}=1+\left(r / R_{1}\right) \sin \theta \cos ^{2}\left(\delta_{p}\right)$, where $\delta_{p}$ is the step's angle of the helix. Likewise, the radius of the curvature of the second bending is $R_{2}$, the length is $\zeta_{2}=\left(R_{2} \phi_{2}\right) / \cos \delta_{p}$, and the metric coefficient is $h_{\zeta_{2}}=1+\left(r / R_{2}\right) \sin \theta \cos ^{2}\left(\delta_{p}\right)$, as shown in Figure 1(a). The total length in this case is given by $\zeta=\zeta_{1}+\zeta_{2}$. The cases for a straight waveguide are obtained by letting $R_{1} \rightarrow \infty$ and $R_{2} \rightarrow \infty$. In the same technique, we can solve practical problems in the case of the flexible hollow waveguide, based on two bendings. Let us assume that the helical waveguide consists of two bendings as shown in Figures 2(a) and 2(b). The Curved-Straight waveguide is shown in Figure 2(a) and the Straight-Curved waveguide is shown in Figure 2(b). These examples are based on the derivation of the helical waveguide with two bendings, where one of the sections is a bending helical waveguide, and the other section is a straight waveguide.

The helical waveguide that consists of three sections (Curved-Straight-Curved waveguide) is shown in Figure 3. The total losses are the sum of the bendings' losses, the dielectric losses, and the losses resulting from the difference in the bendings in the interface between the two bendings. Let us assume that the requirement in this example is that the output power transmission $(\mathrm{T})$ is at least equal to $80 \%(80 \% \leq \mathrm{T} \leq 100 \%)$. This example is based on the derivation of the Curved-Straight waveguide (Figure 2(a)) and the Straight-Curved waveguide (Figure 2(b)). In order to improve the output power transmission of the Curved-Straight waveguide (Figure 2(a)), of the Straight-Curved waveguide (Figure 2(b)), or of the Curved-Straight-Curved waveguide (Figure 3), we have to find the relevant parameters from the results of the output power transmission of the first helical bending.

We start by finding the metric coefficients from the helical transformation of the coordinates. The helical transformation of the coordinates is achieved by two rotations and one translation, and is given in the form:

$$
\left(\begin{array}{l}
X \\
Y \\
Z
\end{array}\right)=\left(\begin{array}{ccc}
\cos \left(\phi_{c}\right) & -\sin \left(\phi_{c}\right) & 0 \\
\sin \left(\phi_{c}\right) & \cos \left(\phi_{c}\right) & 0 \\
0 & 0 & 1
\end{array}\right)\left(\begin{array}{ccc}
1 & 0 & 0 \\
0 & \cos \left(\delta_{p}\right) & -\sin \left(\delta_{p}\right) \\
0 & \sin \left(\delta_{p}\right) & \cos \left(\delta_{p}\right)
\end{array}\right)\left(\begin{array}{c}
r \sin \theta \\
0 \\
r \cos \theta
\end{array}\right)+\left(\begin{array}{c}
R \cos \left(\phi_{c}\right) \\
R \sin \left(\phi_{c}\right) \\
\zeta \sin \left(\delta_{p}\right)
\end{array}\right),
$$

where $\zeta$ is the coordinate along the helix axis, $\mathrm{R}$ is the radius of the cylinder, $\delta_{p}$ is the step's angle of the helix (see Figures (4(a))-(4(b))), and $\phi_{c}=\left(\zeta \cos \left(\delta_{p}\right)\right) / R$. Likewise, $0 \leq r \leq a+\delta_{m}$, where 2a is the internal diameter of the cross-section of the helical waveguide, $\delta_{m}$ is the thickness of the metallic layer, and $\mathrm{d}$ is the thickness of the dielectric layer (see Figure 1(d)). The cross-section of the helical waveguide in the region $0 \leq r \leq a+\delta_{m}$ is 
shown in Figure 1(d), where $\delta_{m}$ is the thickness of the metallic layer, and $\mathrm{d}$ is the thickness of the dielectric layer. Figure 4(a) shows the rotations and translation of the orthogonal system $(\bar{X}, \bar{\zeta}, \bar{Z})$ from point $A$ to the orthogonal system $(\mathrm{X}, \mathrm{Y}, \mathrm{Z})$ at point $K$. Figure 4(b) shows the deployment of the helix depicted in Figure 4(a). The condition for the step's angle $\delta_{p}$ is given according to

$$
\tan \left(\delta_{p}\right) \geq \frac{2\left(a+\delta_{m}\right)}{2 \pi R},
$$

where the internal diameter is denoted as $2 \mathrm{a}$, the thickness of the metallic layer is denoted as $\delta_{m}$, and the radius of the cylinder is denoted as $\mathrm{R}$.

According to Equation (1), the helical transformation of the coordinates becomes

$$
\begin{gathered}
X=(R+r \sin \theta) \cos \left(\phi_{c}\right)+r \sin \left(\delta_{p}\right) \cos \theta \sin \left(\phi_{c}\right), \\
Y=(R+r \sin \theta) \sin \left(\phi_{c}\right)-r \sin \left(\delta_{p}\right) \cos \theta \cos \left(\phi_{c}\right), \\
Z=r \cos \theta \cos \left(\delta_{p}\right)+\zeta \sin \left(\delta_{p}\right),
\end{gathered}
$$

where $\phi_{c}=(\zeta / R) \cos \left(\delta_{p}\right), \mathrm{R}$ is the radius of the cylinder, and (r, $\left.\theta\right)$ are the parameters of the cross-section. Note that $\zeta \sin \left(\delta_{p}\right)=R \phi_{c} \tan \left(\delta_{p}\right)$.

The metric coefficients in the case of the helical waveguide according to Eqs. (3a)-(3c) are:

$$
\begin{gathered}
h_{r}=1 \quad, \quad h_{\theta}=r, \\
h_{\zeta}=\sqrt{\left(1+\frac{r}{R} \sin \theta\right)^{2} \cos ^{2}\left(\delta_{p}\right)+\sin ^{2}\left(\delta_{p}\right)\left(1+\frac{r^{2}}{R^{2}} \cos ^{2} \theta \cos ^{2}\left(\delta_{p}\right)\right)} \\
=\sqrt{1+\frac{2 r}{R} \sin \theta \cos ^{2}\left(\delta_{p}\right)+\frac{r^{2}}{R^{2}} \sin ^{2} \theta \cos ^{2}\left(\delta_{p}\right)+\frac{r^{2}}{R^{2}} \cos ^{2} \theta \cos ^{2}\left(\delta_{p}\right) \sin ^{2}\left(\delta_{p}\right)} \\
\simeq 1+\frac{r}{R} \sin \theta \cos ^{2}\left(\delta_{p}\right) .
\end{gathered}
$$

Note that the third and the fourth terms in the root of the metric coefficient $h_{\zeta}$ are negligible in comparison to the first and the second terms when $(r / R)^{2} \ll 1$.

Further we assume that the derivation is based on an arbitrary value of the step's angle of the helix $\left(\delta_{p}\right)$. The derivation is based on Maxwell's equations for the computation of the EM field and the radiation power density at each point during propagation along a helical waveguide, with a radial dielectric profile. The longitudinal components of the fields are developed into the Fourier-Bessel series. The transverse components of the fields are expressed as a function of the longitudinal components in the Laplace transform domain. Finally, the transverse components of the fields are obtained by using the inverse Laplace transform by the residue method, for an arbitrary value of the step's angle of the helix $\left(\delta_{p}\right)$.

The wave equations for the electric and magnetic field components in the inhomogeneous dielectric medium $\epsilon(r)$ are given by

$$
\nabla^{2} \boldsymbol{E}+\omega^{2} \mu \epsilon \boldsymbol{E}+\nabla\left(\boldsymbol{E} \cdot \frac{\nabla \epsilon}{\epsilon}\right)=0,
$$

and

$$
\nabla^{2} \boldsymbol{H}+\omega^{2} \mu \epsilon \boldsymbol{H}+\frac{\nabla \epsilon}{\epsilon} \times(\nabla \times \boldsymbol{H})=0,
$$

respectively. The transverse dielectric profile $(\epsilon(r))$ is defined as $\epsilon_{0}(1+g(r))$, where $\epsilon_{0}$ represents the vacuum dielectric constant, and $g(r)$ is its profile function in the waveguide. The normalized transverse derivative of the dielectric profile $\left(g_{r}\right)$ is defined as $(1 / \epsilon(r))(\partial \epsilon(r) / \partial r)$.

From the transformation of Eqs. (3a)-(3c) we can derive the Laplacian of the vector $\boldsymbol{E}$ (i.e., $\nabla^{2} \boldsymbol{E}$ ), and obtain the wave equations for the electric and magnetic fields in the inhomogeneous dielectric medium. It is necessary to find the values of $\nabla \cdot \boldsymbol{E}, \nabla(\nabla \cdot \boldsymbol{E}), \nabla \times \boldsymbol{E}$, and $\nabla \times(\nabla \times \boldsymbol{E})$ in order to obtain the value of $\nabla^{2} \boldsymbol{E}$, where $\nabla^{2} \boldsymbol{E}=\nabla(\nabla \cdot \boldsymbol{E})-\nabla \times(\nabla \times \boldsymbol{E})$. All these values are dependent on the metric coefficients $((4 \mathrm{a})-(4 \mathrm{c}))$. 
The $\zeta$ component of $\nabla^{2} \boldsymbol{E}$ is given by

$$
\left(\nabla^{2} \boldsymbol{E}\right)_{\zeta}=\nabla^{2} E_{\zeta}+\frac{2}{R h_{\zeta}^{2}}\left[\sin \theta \frac{\partial}{\partial \zeta} E_{r}+\cos \theta \frac{\partial}{\partial \zeta} E_{\theta}\right]-\frac{1}{R^{2} h_{\zeta}^{2}} E_{\zeta}
$$

where

$$
\nabla^{2} E_{\zeta}=\frac{\partial^{2}}{\partial r^{2}} E_{\zeta}+\frac{1}{r^{2}} \frac{\partial^{2}}{\partial \theta^{2}} E_{\zeta}+\frac{1}{r} \frac{\partial}{\partial r} E_{\zeta}+\frac{1}{h_{\zeta}}\left[\frac{\sin \theta}{R} \frac{\partial}{\partial r} E_{\zeta}+\frac{\cos \theta}{r R} \frac{\partial}{\partial \theta} E_{\zeta}+\frac{1}{h_{\zeta}} \frac{\partial^{2}}{\partial \zeta^{2}} E_{\zeta}\right] .
$$

The longitudinal components of the wave Equations (5a) and (5b) are obtained by deriving the following terms

$$
\left[\nabla\left(\boldsymbol{E} \cdot \frac{\nabla \epsilon}{\epsilon}\right)\right]_{\zeta}=\frac{1}{h_{\zeta}} \frac{\partial}{\partial \zeta}\left[E_{r} g_{r}\right]
$$

and

$$
\left[\frac{\nabla \epsilon}{\epsilon} \times(\nabla \times \boldsymbol{H})\right]_{\zeta}=j \omega \epsilon\left[\frac{\nabla \epsilon}{\epsilon} \times \boldsymbol{E}\right]_{\zeta}=j \omega \epsilon g_{r} E_{\theta}
$$

The longitudinal components of the wave Equations (5a) and (5b) are then written in the form

$$
\begin{gathered}
\left(\nabla^{2} \boldsymbol{E}\right)_{\zeta}+k^{2} E_{\zeta}+\frac{1}{h_{\zeta}} \frac{\partial}{\partial \zeta}\left(E_{r} g_{r}\right)=0 \\
\left(\nabla^{2} \boldsymbol{H}\right)_{\zeta}+k^{2} H_{\zeta}+j \omega \epsilon g_{r} E_{\theta}=0
\end{gathered}
$$

where $\left(\nabla^{2} \mathbf{E}\right)_{\zeta}$, for instance, is given in Equation (6). The local wave number parameter is $k=\omega \sqrt{\mu \epsilon(r)}=$ $k_{0} \sqrt{1+g(r)}$, where the free-space wave number is $k_{0}=\omega \sqrt{\mu_{0} \epsilon_{0}}$.

The transverse Laplacian operator is defined as

$$
\nabla_{\perp}^{2} \equiv \nabla^{2}-\frac{1}{h_{\zeta}^{2}} \frac{\partial^{2}}{\partial \zeta^{2}}
$$

The Laplace transform

$$
\tilde{a}(s)=\mathcal{L}\{a(\zeta)\}=\int_{\zeta=0}^{\infty} a(\zeta) e^{-s \zeta} d \zeta
$$

is applied on the $\zeta$-dimension, where $a(\zeta)$ represents any $\zeta$-dependent variables, where $\zeta=\left(R \phi_{c}\right) / \cos \left(\delta_{p}\right)$.

The longitudinal components of the fields $\left(E_{\zeta}, H_{\zeta}\right)$ are developed into Fourier - Bessel series as follows :

$$
\begin{aligned}
& \tilde{E}_{\zeta}(s)=\sum_{n^{\prime}} \sum_{m^{\prime}}\left[A_{n^{\prime} m^{\prime}}(s) \cos \left(n^{\prime} \theta\right)+B_{n^{\prime} m^{\prime}}(s) \sin \left(n^{\prime} \theta\right)\right] J_{n^{\prime}}\left(P_{n^{\prime} m^{\prime}} \frac{r}{a}\right) \\
& \tilde{H}_{\zeta}(s)=\sum_{n^{\prime}} \sum_{m^{\prime}}\left[C_{n^{\prime} m^{\prime}}(s) \cos \left(n^{\prime} \theta\right)+D_{n^{\prime} m^{\prime}}(s) \sin \left(n^{\prime} \theta\right)\right] J_{n^{\prime}}\left(P_{n^{\prime} m^{\prime}}^{\prime} \frac{r}{a}\right)
\end{aligned}
$$

where $P_{n m}$ and $P_{n m}^{\prime}$ are the mth roots of the equations $J_{n}(x)=0$ and $d J_{n}(x) / d x=0$, respectively.

By substituting Equation (6) into Equation (10) and by using the Laplace transform (13), the longitudinal components of the wave equations (Equations (10)-(11)) are described in the Laplace transform domain, as coupled wave equations. The transverse fields for the first section of the helical hollow waveguide with two bendings at $\zeta=\zeta_{1}$ are obtained directly from the Maxwell equations, and by using the Laplace transform (13). The transverse fields for the first section of the helical hollow waveguide are dependent only on the longitudinal components of the fields and as function of the step's angle $\left(\delta_{p}\right)$ of the helix, as follows:

$$
\tilde{E}_{r_{1}}(s)=\frac{1}{s^{2}+k^{2} h_{\zeta_{1}}^{2}}\left\{-\frac{j \omega \mu_{0}}{r}\left[\frac{r}{R_{1}} \cos \theta \cos ^{2}\left(\delta_{p}\right) \tilde{H}_{\zeta_{1}}+h_{\zeta_{1}} \frac{\partial}{\partial \theta} \tilde{H}_{\zeta_{1}}\right] h_{\zeta_{1}}\right.
$$




$$
\begin{gathered}
\left.+s\left[\frac{\sin \theta}{R_{1}} \cos ^{2}\left(\delta_{p}\right) \tilde{E}_{\zeta_{1}}+h_{\zeta_{1}} \frac{\partial}{\partial r} \tilde{E}_{\zeta_{1}}\right]+s E_{r_{0}}-j \omega \mu_{0} H_{\theta_{0}} h_{\zeta_{1}}\right\} \\
\tilde{E}_{\theta_{1}}(s)=\frac{1}{s^{2}+k^{2} h_{\zeta_{1}}^{2}}\left\{\frac{s}{r}\left[\frac{r}{R_{1}} \cos \theta \cos ^{2}\left(\delta_{p}\right) \tilde{E}_{\zeta_{1}}+h_{\zeta_{1}} \frac{\partial}{\partial \theta} \tilde{E}_{\zeta_{1}}\right]\right. \\
\left.+j \omega \mu_{0} h_{\zeta_{1}}\left[\frac{\sin \theta}{R_{1}} \cos ^{2}\left(\delta_{p}\right) \tilde{H}_{\zeta_{1}}+h_{\zeta_{1}} \frac{\partial}{\partial r} \tilde{H}_{\zeta_{1}}\right]+s E_{\theta_{0}}+j \omega \mu_{0} H_{r_{0}} h_{\zeta_{1}}\right\} \\
\tilde{H}_{r_{1}}(s)=\frac{1}{s^{2}+k^{2} h_{\zeta_{1}}^{2}}\left\{\frac{j \omega \epsilon}{r}\left[\frac{r}{R_{1}} \cos \theta \cos ^{2}\left(\delta_{p}\right) \tilde{E}_{\zeta_{1}}+h_{\zeta_{1}} \frac{\partial}{\partial \theta} \tilde{E}_{\zeta_{1}}\right] h_{\zeta_{1}}\right. \\
\left.+s\left[\frac{\sin \theta}{R_{1}} \cos ^{2}\left(\delta_{p}\right) \tilde{H}_{\zeta_{1}}+h_{\zeta_{1}} \frac{\partial}{\partial r} \tilde{H}_{\zeta_{1}}\right]+s H_{r_{0}}+j \omega \epsilon E_{\theta_{0}} h_{\zeta_{1}}\right\} \\
\tilde{H}_{\theta_{1}}(s)=\frac{1}{s^{2}+k^{2} h_{\zeta_{1}}^{2}}\left\{\frac{s}{r}\left[\frac{r}{R_{1}} \cos \theta \cos ^{2}\left(\delta_{p}\right) \tilde{H}_{\zeta_{1}}+h_{\zeta_{1}} \frac{\partial}{\partial \theta} \tilde{H}_{\zeta_{1}}\right]\right. \\
\left.-j \omega \epsilon h_{\zeta_{1}}\left[\frac{\sin \theta}{R_{1}} \cos ^{2}\left(\delta_{p}\right) \tilde{E}_{\zeta_{1}}+h_{\zeta_{1}} \frac{\partial}{\partial r} \tilde{E}_{\zeta_{1}}\right]+s H_{\theta_{0}}-j \omega \epsilon E_{r_{0}} h_{\zeta_{1}}\right\}
\end{gathered}
$$

where $\zeta_{1}$ is the coordinate along the first section of the helical axis, $R_{1}$ is the radius of the cylinder of the first section of the helical axis, where the metric coefficient is $h_{\zeta_{1}}=1+\left(r / R_{1}\right) \sin \theta \cos ^{2}\left(\delta_{p}\right)$.

2.1 The Elements of the Boundary Conditions' Vectors in the Entrance of the First Section of the Helical Waveguide, and the Fields at $\zeta=\zeta_{1}$

The separation of variables is obtained by using the orthogonal-relations. Thus the algebraic equations $(n \neq 0)$ are given by

$$
\begin{aligned}
& \alpha_{n}{ }^{(1)} A_{n}+\beta_{n}{ }^{(1)} D_{n}=\frac{1}{\pi}{\widehat{(B C 1)_{n}}}, \\
& \alpha_{n}{ }^{(2)} B_{n}+\beta_{n}{ }^{(2)} C_{n}=\frac{1}{\pi} \widehat{(B C 2)_{n}}, \\
& \beta_{n}{ }^{(3)} B_{n}+\alpha_{n}{ }^{(3)} C_{n}=\frac{1}{\pi} \widehat{(B C 3)_{n}}, \\
& \beta_{n}{ }^{(4)} A_{n}+\alpha_{n}{ }^{(4)} D_{n}=\frac{1}{\pi} \widehat{(B C 4)}_{n} .
\end{aligned}
$$

Further we assume $n^{\prime}=\mathrm{n}=1$. The elements $\left(\alpha_{n}{ }^{(1)}, \beta_{n}{ }^{(1)}\right.$, etc), on the left side of (16a) for $\mathrm{n}=1$ are given for an arbitrary value of the step's angle $\left(\delta_{p}\right)$ for the first section of the helical waveguide and the elements of the matrices $\left(G_{00}^{(1) m m^{\prime}}\right.$, etc.) are given in Appendix A.

The elements of the boundary conditions' vectors on the right side in Equations (16a)-(16d) are changed at the entrance of every section of the helical waveguide with two bendings. These elements are given in conjunction with the excitation of every section, as follows:

$$
\begin{aligned}
& \widehat{(B C 1)_{1}}=\int_{0}^{2 \pi} \int_{0}^{a}(B C 1) \cos (\theta) J_{1}\left(P_{1 m} r / a\right) r d r d \theta, \\
& \widehat{(B C 2)_{1}}=\int_{0}^{2 \pi} \int_{0}^{a}(B C 2) \sin (\theta) J_{1}\left(P_{1 m} r / a\right) r d r d \theta, \\
& \widehat{(B C 3)_{1}}=\int_{0}^{2 \pi} \int_{0}^{a}(B C 3) \cos (\theta) J_{1}\left(P_{1 m}^{\prime} r / a\right) r d r d \theta, \\
& \widehat{(B C 4)_{1}}=\int_{0}^{2 \pi} \int_{0}^{a}(B C 4) \sin (\theta) J_{1}\left(P_{1 m}^{\prime} r / a\right) r d r d \theta .
\end{aligned}
$$


In the case of the $T E M_{00}$ mode in excitation for the first section of the helical waveguide with two bendings (Figure 1(a)), the elements of the boundary conditions' vectors are obtained, where:

$$
\begin{aligned}
B C 1= & B C 2=j \omega \mu_{0} H_{\theta_{0}}^{+} s g_{r} h_{\zeta_{1}}^{2}+\frac{2}{R_{1}} h_{\zeta_{1}} \sin \theta\left(j \omega \mu_{0} H_{\theta_{0}}^{+} s+k^{2} E_{r_{0}}^{+} h_{\zeta_{1}}\right) \\
& +\frac{2}{R_{1}} h_{\zeta_{1}} \cos \theta\left(-j \omega \mu_{0} H_{r_{0}}^{+} s+k^{2} E_{\theta_{0}}^{+} h_{\zeta_{1}}\right)+k^{2} h_{\zeta_{1}}^{3} E_{r_{0}}^{+} g_{r}, \\
B C 3= & B C 4=-j \omega \epsilon E_{\theta_{0}}^{+} s g_{r} h_{\zeta_{1}}^{2}+\frac{2}{R_{1}} h_{\zeta_{1}} \sin \theta\left(k^{2} h_{\zeta_{1}} H_{r_{0}}^{+}-j \omega \epsilon s E_{\theta_{0}}^{+}\right) \\
& +\frac{2}{R_{1}} h_{\zeta_{1}} \cos \theta\left(k^{2} h_{\zeta_{1}} H_{\theta_{0}}^{+}+j \omega \epsilon s E_{r_{0}}^{+}\right)+k^{2} h_{\zeta_{1}}^{3} H_{r_{0}}^{+} g_{r} .
\end{aligned}
$$

From the elements of the boundary conditions (e.g., $\left.\widehat{(B C 2)_{1}}\right)$ at $\zeta_{1}=0^{+}$on the right side in (16b), the boundary conditions at $\zeta_{1}=0^{+}$for $T E M_{00}$ mode in excitation become to:

$$
\begin{gathered}
\widehat{(B C 2)_{1}}=2 \pi\left\{\int_{0}^{a} Q(r)(k(r)+j s) k(r) J_{1 m}\left(P_{1 m} r / a\right) r d r\right\} \delta_{1 n}+\frac{4 j s \pi}{R_{1}^{2}} \cos ^{2}\left(\delta_{p}\right)\left\{\int_{0}^{a} Q(r) k(r) J_{1 m}\left(P_{1 m} r / a\right) r^{2} d r\right\} \delta_{1 n} \\
+\frac{9 \pi}{2 R_{1}^{2}} \cos ^{4}\left(\delta_{p}\right)\left\{\int_{0}^{a} Q(r) k^{2}(r) J_{1 m}\left(P_{1 m} r / a\right) r^{3} d r\right\} \delta_{1 n}+\frac{3 j s \pi}{2 R_{1}^{2}} \cos ^{4}\left(\delta_{p}\right)\left\{\int_{0}^{a} Q(r) k(r) J_{1 m}\left(P_{1 m} r / a\right) r^{3} d r\right\} \delta_{1 n} \\
+\frac{8 \pi}{R_{1}^{2}} \cos ^{2}\left(\delta_{p}\right)\left\{\int_{0}^{a} Q(r) k^{2}(r) J_{1 m}\left(P_{1 m} r / a\right) r^{2} d r\right\} \delta_{1 n}
\end{gathered}
$$

where

$$
Q(r)=\frac{E_{0}}{n_{c}(r)+1} g_{r} \exp \left(-\left(r / w_{o}\right)^{2}\right) .
$$

Similarly, the remaining elements of the boundary conditions at $\zeta=0^{+}$are obtained. The matrix system of the Equations (16a)-(16d) is solved to obtain the coefficients $\left(A_{1}, B_{1}\right.$, etc).

According to the Gaussian beams (Yariv A., 1985) the parameter $w_{0}$ is the minimum spot-size at the plane $\mathrm{z}=0$, and the electric field at the plane $\mathrm{z}=0$ is given by $E=E_{0} \exp \left[-\left(r / w_{o}\right)^{2}\right]$. The modes excited at $\zeta=0$ in the waveguide by the conventional $\mathrm{CO}_{2}$ laser IR radiation $(\lambda=10.6 \mu \mathrm{m})$ are closer to the TEM polarization of the laser radiation. The $T E M_{00}$ mode is the fundamental and most important mode. This means that a cross-section of the beam has a Gaussian intensity distribution. The relation between the electric and magnetic fields (Yariv A., 1985) is given by $E / H=\sqrt{\mu_{0} / \epsilon_{0}} \equiv \eta_{0}$, where $\eta_{0}$ is the intrinsic wave impedance. Suppose that the electric field is parallel to the y-axis. Thus the components of $E_{y}$ and $H_{x}$ are written by the fields $E_{y}=E_{0} \exp \left[-\left(r / w_{o}\right)^{2}\right]$ and $H_{x}=-\left(E_{0} / \eta_{0}\right) \exp \left[-\left(r / w_{o}\right)^{2}\right]$.

After a Gaussian beam passes through a lens and before it enters to the waveguide, the waist cross-sectional diameter $\left(2 w_{0}\right)$ can then be calculated approximately for a parallel incident beam by means of $w_{0}=\lambda /(\pi \theta) \simeq(\mathrm{f}$ $\lambda) /(\pi \mathrm{w})$. This approximation is justified if the parameter $w_{0}$ is much larger than the wavelength $\lambda$. The parameter of the waist cross-sectional diameter $\left(2 w_{0}\right)$ is taken into account in our method, instead of the focal length of the lens (f). The initial fields at $\zeta=0^{+}$are formulated by using the Fresnel coefficients of the transmitted fields (Baden Fuller A. J., 1969) as follows

$$
\begin{gathered}
E_{r_{0}}^{+}\left(r, \theta, \zeta=0^{+}\right)=T_{E}(r)\left(E_{0} e^{-\left(r / w_{o}\right)^{2}} \sin \theta\right), \\
E_{\theta_{0}}^{+}\left(r, \theta, \zeta=0^{+}\right)=T_{E}(r)\left(E_{0} e^{-\left(r / w_{o}\right)^{2}} \cos \theta\right), \\
H_{r_{0}}^{+}\left(r, \theta, \zeta=0^{+}\right)=-T_{H}(r)\left(\left(E_{0} / \eta_{0}\right) e^{-\left(r / w_{o}\right)^{2}} \cos \theta\right), \\
H_{\theta_{0}}^{+}\left(r, \theta, \zeta=0^{+}\right)=T_{H}(r)\left(\left(E_{0} / \eta_{0}\right) e^{-\left(r / w_{o}\right)^{2}} \sin \theta\right),
\end{gathered}
$$

where $E_{\zeta_{0}}^{+}(r)=H_{\zeta_{0}}^{+}=0, T_{E}(r)=2 /[n(r)+1], T_{H}(r)=2 n(r) /[n(r)+1]$, and $n(r)=\left(\epsilon_{r}(r)\right)^{1 / 2}$. The index of refraction is denoted by $n(r)$. 
The output transverse components of the fields of the helical waveguide are dependent also on the step's angle of the helix $\left(\delta_{p}\right)$ and the radius of the cylinder of the helix for the first section $\left(R_{1}\right)$. The output transverse components of the fields are finally expressed in a form of transfer matrix functions for the first bending of the helical waveguide as follows:

$$
\begin{gathered}
E_{r_{1}}\left(r, \theta, \zeta_{1}\right)=E_{r 0}^{+}(r) e^{-j k h_{\zeta_{1}} \zeta}-\frac{j \omega \mu_{0}}{R_{1}} h_{\zeta_{1}} \cos ^{2} \theta \cos ^{2}\left(\delta_{p}\right) \sum_{m^{\prime}} C_{S 1}^{m^{\prime}}\left(\zeta_{1}\right) J_{1}(\psi) \\
-\frac{j \omega \mu_{0}}{R_{1}} h_{\zeta_{1}} \sin \theta \cos \theta \cos ^{2}\left(\delta_{p}\right) \sum_{m^{\prime}} D_{S 1}^{m^{\prime}}\left(\zeta_{1}\right) J_{1}(\psi)+\frac{j \omega \mu_{0}}{r} h_{\zeta_{1}}^{2} \sin \theta \sum_{m^{\prime}} C_{S 1}^{m^{\prime}}\left(\zeta_{1}\right) J_{1}(\psi) \\
-\frac{j \omega \mu_{0}}{r} h_{\zeta_{1}}^{2} \cos \theta \sum_{m^{\prime}} D_{S 1}^{m^{\prime}}\left(\zeta_{1}\right) J_{1}(\psi)+\frac{1}{R_{1}} \sin \theta \cos \theta \cos ^{2}\left(\delta_{p}\right) \sum_{m^{\prime}} A_{S 2}^{m^{\prime}}\left(\zeta_{1}\right) J_{1}(\xi) \\
+\frac{1}{R_{1}} \sin ^{2} \theta \cos ^{2}\left(\delta_{p}\right) \sum_{m^{\prime}} B_{S 2}^{m^{\prime}}\left(\zeta_{1}\right) J_{1}(\xi)+h_{\zeta_{1}} \cos \theta \sum_{m^{\prime}} A_{S 2}^{m^{\prime}}\left(\zeta_{1}\right) \frac{d J_{1}}{d r}(\xi) \\
+h_{\zeta_{1}} \sin \theta \sum_{m^{\prime}} B_{S 2}^{m^{\prime}}\left(\zeta_{1}\right) \frac{d J_{1}}{d r}(\xi)
\end{gathered}
$$

where $h_{\zeta_{1}}=1+\left(r / R_{1}\right) \sin \theta \cos ^{2}\left(\delta_{p}\right), \quad \mathrm{R}$ is the radius of the cylinder, $\delta_{p}$ is the the step's angle, $\psi=\left[P_{1 m^{\prime}}^{\prime}(r / a)\right]$ and $\xi=\left[P_{1 m^{\prime}}(r / a)\right]$. The coefficients are given in the above equation, for instance

$$
A_{S 1}^{m^{\prime}}\left(\zeta_{1}\right)=\mathcal{L}^{-1}\left\{\frac{A_{1 m^{\prime}}(s)}{s^{2}+k^{2}(r) h_{\zeta_{1}}^{2}}\right\}, \quad A_{S 2}^{m^{\prime}}\left(\zeta_{1}\right)=\mathcal{L}^{-1}\left\{\frac{s A_{1 m^{\prime}}(s)}{s^{2}+k^{2}(r) h_{\zeta_{1}}^{2}}\right\},
$$

where

$$
m^{\prime}=1, \ldots N, \quad 3 \leq N \leq 50 .
$$

Similarly, the other transverse components of the output fields are obtained. The first fifty roots (zeros) of the equations $J_{1}(x)=0$ and $d J_{1}(x) / d x=0$ may be found in tables (Olver F. W. J., 1960) (Jahnke E., and Emde F., 1945).

2.2 The Elements of the Boundary Conditions' Vectors in the Entrance of the Second Section of the Helical Waveguide at $\zeta=\zeta_{1}$, and the Transverse Fields at $\zeta=\zeta_{1}+\zeta_{2}$

The elements of the boundary conditions' vectors (17a)-(17d) in the case of the $T E M_{00}$ mode in excitation for the second section of the helical waveguide with two bendings are obtained from the algebraic system of Equations (16a)-(16d) for $n=1$, as follows:

$$
\begin{aligned}
B C 1= & B C 2=j \omega \mu_{0} H_{\theta}\left(\zeta=\zeta_{1}\right) s g_{r} h_{\zeta_{2}}^{2}+\frac{2}{R_{2}} \sin \theta h_{\zeta_{2}}\left(j \omega \mu_{0} H_{\theta}\left(\zeta=\zeta_{1}\right) s+k^{2} E_{r}\left(\zeta=\zeta_{1}\right) h_{\zeta_{2}}\right) \\
& +\frac{2}{R_{2}} \cos \theta h_{\zeta_{2}}\left(-j \omega \mu_{0} H_{r}\left(\zeta=\zeta_{1}\right) s+k^{2} E_{\theta}\left(\zeta=\zeta_{1}\right) h_{\zeta_{2}}\right)+k^{2} h_{\zeta_{2}}^{3} E_{r}\left(\zeta=\zeta_{1}\right) g_{r}, \\
B C 3= & B C 4=-j \omega \epsilon E_{\theta}\left(\zeta=\zeta_{1}\right) s g_{r} h_{\zeta_{2}}^{2}+\frac{2}{R_{2}} \sin \theta h_{\zeta_{2}}\left(k^{2} h_{\zeta_{2}} H_{r}\left(\zeta=\zeta_{1}\right)-j \omega \epsilon s E_{\theta}\left(\zeta=\zeta_{1}\right)\right) \\
& +\frac{2}{R_{2}} \cos \theta h_{\zeta_{2}}\left(k^{2} h_{\zeta_{2}} H_{\theta}\left(\zeta=\zeta_{1}\right)+j \omega \epsilon s E_{r}\left(\zeta=\zeta_{1}\right)\right)+k^{2} h_{\zeta_{2}}^{3} H_{r}\left(\zeta=\zeta_{1}\right) g_{r} .
\end{aligned}
$$

Equations (23a)-(23b) are different from Equations (18a)-(18b) by the initial fields. The initial field, for instance, at the entrance of the first section of the helical waveguide at $\zeta=0^{+}$is denoted as $E_{r_{0}}^{+}$according to (20a), but the initial field of the second section of the helical waveguide in the entrance, at $\zeta=\zeta_{1}$, is denoted as $E_{r}\left(\zeta=\zeta_{1}\right)$, according to (21). Similarly, the remaining initial fields are obtained.

These expressions (23a), (23b) are dependent on the radius of the cylinder of the helix of the first section $\left(R_{1}\right)$ and the second section $\left(R_{2}\right)$ of the helical waveguide with two bendings $\left(R_{1} \neq R_{2}\right)$. Note that these expressions 
are given also for an arbitrary values of the step's angle (and not for small values of the step angles). Actually, these expressions ((23a), (23b)) of the elements of the boundary conditions's vectors of the second section of the helical waveguide with two bendings consist of all the information at the output fields of the first section (the Bessel-equations, the dielectric profile $\mathrm{g}(\mathrm{r})$, the transverse derivative $g_{r}(r)$, the parameters of the cross-section (r, $\theta$ ), and the propagation constants $\beta_{n m}$ and $\beta_{n m}^{\prime}$ of the TM and TE modes of the hollow waveguide, respectively).

The expression $\left[1 /\left(s^{2}+k^{2} h_{\zeta_{1}}^{2}\right)\right]\left(s E_{r_{0}}^{+}-j \omega \mu_{0} H_{\theta_{0}}^{+} h_{\zeta_{1}}\right)$ in Equation (15a) for the first section of the helical waveguide with two bendings depends on the inital fields $E_{r_{0}}^{+}(20 \mathrm{a})$ and $H_{\theta_{0}}^{+}(20 \mathrm{~d})$. In the same principle we have the expression $\left[1 /\left(s^{2}+k^{2} h_{\zeta_{2}}^{2}\right)\right]\left(s E_{r}\left(\zeta=\zeta_{1}\right)-j \omega \mu_{0} H_{\theta}\left(\zeta=\zeta_{1}\right) h_{\zeta_{2}}\right)$ for the second section of the helical waveguide with two bendings, that depends on the output fields $E_{r}\left(\zeta=\zeta_{1}\right)$ and $H_{\theta}\left(\zeta=\zeta_{1}\right)\left(\right.$ e.g., (21)) at $\zeta=\zeta_{1}$ of the first section of the helical waveguide with two bendings.

The values of the input fields of the second section of the helical waveguide with two bendings are functions of the parameters of the first section $\left(R_{1}, \zeta_{1}\right.$, and $\left.h_{\zeta_{1}}\right)$ and the second section $\left(R_{2}, \zeta_{2}\right.$, and $\left.h_{\zeta_{2}}\right)$ as shown in Figure 1(a). The expressions are functions of the cross-section $(\mathrm{r}, \theta)$, the sum of the modes and the old coefficients of the first section of the helical waveguide with two bendings.

The output transverse components of the fields of the helical waveguide are dependent also on the step's angle of the helix $\left(\delta_{p}\right)$ and the radius of the cylinder of the helix for the first section $\left(R_{1}\right)$ and for the second section $\left(R_{2}\right)$. The output transverse components of the fields are finally expressed in a form of transfer matrix functions as follows

$$
\begin{aligned}
& E_{r}\left(r, \theta, \zeta=\zeta_{1}+\zeta_{2}\right)=E_{r_{0}}^{+} e^{-j k(r)\left(h_{\zeta_{1}} \zeta_{1}+h_{\zeta_{2}} \zeta_{2}\right)}+\cos \left(k h_{\zeta_{2}} \zeta_{2}\right)\left(-\frac{j \omega \mu_{0}}{R_{1}} h_{\zeta_{1}} \cos ^{2} \theta \cos ^{2}\left(\delta_{p}\right) \sum_{m^{\prime}} C_{S 1}^{m^{\prime}}\left(\zeta_{1}\right) J_{1}(\psi)\right. \\
& -\frac{j \omega \mu_{0}}{R_{1}} h_{\zeta_{1}} \sin \theta \cos \theta \cos ^{2}\left(\delta_{p}\right) \sum_{m^{\prime}} D_{S 1}^{m^{\prime}}\left(\zeta_{1}\right) J_{1}(\psi)+\frac{j \omega \mu_{0}}{r} h_{\zeta_{1}}^{2} \sin \theta \sum_{m^{\prime}} C_{S 1}^{m^{\prime}}\left(\zeta_{1}\right) J_{1}(\psi) \\
& -\frac{j \omega \mu_{0}}{r} h_{\zeta_{1}}^{2} \cos \theta \sum_{m^{\prime}} D_{S 1}^{m^{\prime}}\left(\zeta_{1}\right) J_{1}(\psi)+\frac{1}{R_{1}} \sin \theta \cos \theta \cos ^{2}\left(\delta_{p}\right) \sum_{m^{\prime}} A_{S 2}^{m^{\prime}}\left(\zeta_{1}\right) J_{1}(\xi)+\frac{1}{R_{1}} \sin ^{2} \theta \cos ^{2}\left(\delta_{p}\right) \sum_{m^{\prime}} B_{S 2}^{m^{\prime}}\left(\zeta_{1}\right) J_{1}(\xi) \\
& \left.+h_{\zeta 1} \cos \theta \sum_{m^{\prime}} A_{S 2}^{m^{\prime}} \frac{d J_{1}}{d r}(\xi)+h_{\zeta 1} \sin \theta \sum_{m^{\prime}} B_{S 2}^{m^{\prime}} \frac{d J_{1}}{d r}(\xi)\right) \\
& -\frac{\sin \left(k h_{\zeta_{2}} \zeta_{2}\right)}{k h_{\zeta_{2}}}\left(j \omega \mu_{0} h_{\zeta_{2}}\right)\left(-\frac{j \omega \epsilon}{R_{1}} h_{\zeta_{1}} \sin \theta \cos \theta \cos ^{2}\left(\delta_{p}\right) \sum_{m^{\prime}} A_{S 1}^{m^{\prime}}\left(\zeta_{1}\right) J_{1}(\xi)-\frac{j \omega \epsilon}{R_{1}} h_{\zeta_{1}} \sin ^{2} \theta \cos ^{2}\left(\delta_{p}\right) \sum_{m^{\prime}} B_{S 1}^{m^{\prime}}\left(\zeta_{1}\right) J_{1}(\xi)\right. \\
& -j \omega \epsilon h_{\zeta_{1}}^{2} \cos \theta \sum_{m^{\prime}} A_{S 1}^{m^{\prime}} \frac{d J_{1}}{d r}(\xi)-j \omega \epsilon h_{\zeta_{1}}^{2} \sin \theta \sum_{m^{\prime}} B_{S 1}^{m^{\prime}} \frac{d J_{1}}{d r}(\xi)+\frac{\cos ^{2} \theta}{R_{1}} \cos ^{2}\left(\delta_{p}\right) \sum_{m^{\prime}} C_{S 2}^{m^{\prime}}\left(\zeta_{1}\right) J_{1}(\psi) \\
& \left.+\frac{1}{R_{1}} \sin \theta \cos \theta \cos ^{2}\left(\delta_{p}\right) \sum_{m^{\prime}} D_{S 2}^{m^{\prime}}\left(\zeta_{1}\right) J_{1}(\psi)-\frac{1}{r} h_{\zeta_{1}} \sin \theta \sum_{m^{\prime}} C_{S 2}^{m^{\prime}}\left(\zeta_{1}\right) J_{1}(\psi)+\frac{1}{r} h_{\zeta_{1}} \cos \theta \sum_{m^{\prime}} D_{S 2}^{m^{\prime}}\left(\zeta_{1}\right) J_{1}(\psi)\right) \\
& -\frac{j \omega \mu_{0}}{R_{2}} h_{\zeta_{2}} \cos ^{2} \theta \cos ^{2}\left(\delta_{p}\right) \sum_{m^{\prime}} C_{S 1}^{(2) m^{\prime}}\left(\zeta_{2}\right) J_{1}(\psi)-\frac{j \omega \mu_{0}}{R_{2}} h_{\zeta_{2}} \sin \theta \cos \theta \cos ^{2}\left(\delta_{p}\right) \sum_{m^{\prime}}{ }_{S 1}^{(2) m^{\prime}}\left(\zeta_{2}\right) J_{1}(\psi) \\
& +\frac{j \omega \mu_{0}}{r} h_{\zeta_{2}}^{2} \sin \theta \sum_{m^{\prime}}^{(2) m^{\prime}}\left(\zeta_{2}\right) J_{1}(\psi)-\frac{j \omega \mu_{0}}{r} h_{\zeta_{2}}^{2} \cos \theta \sum_{m^{\prime}} D_{S 1}^{(2) m^{\prime}}\left(\zeta_{2}\right) J_{1}(\psi) \\
& +\frac{1}{R_{2}} \sin \theta \cos \theta \cos ^{2}\left(\delta_{p}\right) \sum_{m^{\prime}} A_{S 2}^{(2) m^{\prime}}\left(\zeta_{2}\right) J_{1}(\xi)+\frac{1}{R_{2}} \sin ^{2} \theta \cos ^{2}\left(\delta_{p}\right) \sum_{m^{\prime}} B_{S 2}^{(2) m^{\prime}}\left(\zeta_{2}\right) J_{1}(\xi) \\
& +h_{\zeta_{2}} \cos \theta \sum_{m^{\prime}} A_{S 2}^{(2) m^{\prime}}\left(\zeta_{2}\right) \frac{d J_{1}}{d r}(\xi)+h_{\zeta_{2}} \sin \theta \sum_{m^{\prime}} B_{S 2}^{(2) m^{\prime}}\left(\zeta_{2}\right) \frac{d J_{1}}{d r}(\xi)
\end{aligned}
$$

where $\psi=\left[P_{1 m^{\prime}}^{\prime}(r / a)\right]$ and $\xi=\left[P_{1 m^{\prime}}(r / a)\right], h_{\zeta_{1}}=1+\left(r / R_{1}\right) \sin \theta \cos ^{2}\left(\delta_{p}\right)$, and $h_{\zeta_{2}}=1+\left(r / R_{2}\right) \sin \theta \cos ^{2}\left(\delta_{p}\right)$. 
The new coefficients are given in the above equation, for instance

$$
A_{S 1}^{(2) m^{\prime}}\left(\zeta_{2}\right)=\mathcal{L}^{-1}\left[\frac{A_{1 m^{\prime}}^{(2)}(s)}{s^{2}+k^{2}(r) h_{\zeta_{2}}^{2}}\right], \quad A_{S 2}^{(2) m^{\prime}}\left(\zeta_{2}\right)=\mathcal{L}^{-1}\left[\frac{s A_{1 m^{\prime}}^{(2)}(s)}{s^{2}+k^{2}(r) h_{\zeta_{2}}^{2}}\right],
$$

where

$$
m^{\prime}=1, \ldots N, \quad 3 \leq N \leq 50 .
$$

Similarly, the other output transverse components of the fields are obtained, in a form of transfer matrix functions. The general solutions of the output transverse components of the fields (e.g., Equation (24)) at $\zeta=\zeta_{1}+\zeta_{2}$ are dependent on the initial fields at $\zeta=\zeta_{1}$ along the total length $\zeta=\zeta_{1}+\zeta_{2}$, where $\zeta_{1}=\left(R_{1} \phi_{1}\right) / \cos \delta_{p}$ and $\zeta_{2}=\left(R_{2} \phi_{2}\right) / \cos \delta_{p}$. Note that the main expression of the solution of the output transverse components of the fields (e.g., Equation (21)) is proportional to $E_{r_{0}}^{+} \exp \left[-j k(r) h_{\zeta_{1}} \zeta_{1}\right]$. In the same principle, the main expression for the second section of the helical waveguide with two bendings (e.g., Equation (24)) is proportional to $E_{r_{0}}^{+} \exp \left[-j k(r)\left(h_{\zeta_{1}} \zeta_{1}+h_{\zeta_{2}} \zeta_{2}\right)\right]$.

These above solutions (e.g., (24)) consist of the sum of the principle expression and other expressions. The principle expression is multiplication of the initial field at $\zeta=0^{+}(20 \mathrm{a})$ with the exponent $\left[\exp \left[-j k(r)\left(h_{\zeta_{1}} \zeta_{1}+\right.\right.\right.$ $\left.\left.\left.h_{\zeta_{2}} \zeta_{2}\right)\right]\right]$. All the above derivation is introduced in the case of the helical waveguide with two bendings in the same direction.

The inverse Laplace transform is performed in this study by a direct numerical integration in the Laplace transform domain by the residue method, as follows

$$
f(\zeta)=\mathcal{L}^{-1}[\tilde{f}(s)]=\frac{1}{2 \pi j} \int_{\sigma-j_{\infty}}^{\sigma+j_{\infty}} \tilde{f}(s) e^{s \zeta} d s=\sum_{n} \operatorname{Res}\left[e^{s \zeta} \tilde{f}(s) ; S_{n}\right]
$$

By using the inverse Laplace transform (26) we can compute the output transverse components in the real plane and the output power density at each point at $\zeta=\left(\mathrm{R} \phi_{c}\right) / \cos \left(\delta_{p}\right)$. The integration path in the right side of the Laplace transform domain includes all the singularities according to Equation (26). All the points $S_{n}$ are the poles of $\tilde{f}(s)$ and $\operatorname{Res}\left[e^{s \zeta} \tilde{f}(s) ; S_{n}\right]$ represent the residue of the function in a specific pole. According to the residue method, two dominant poles for the helical waveguide are given by

$$
s= \pm j k(r) h_{\zeta}= \pm j k(r)\left(1+\frac{r}{R} \sin \theta \cos ^{2}\left(\delta_{p}\right)\right) .
$$

Finally, knowing all the transverse components, the $\zeta$ component of the average-power density Poynting vector is given by

$$
S_{a v}=\frac{1}{2} \operatorname{Re}\left\{E_{r} H_{\theta}{ }^{*}-E_{\theta} H_{r}^{*}\right\} \quad
$$

where the asterisk indicates the complex conjugate.

The total average-power transmitted along the guide in the $\zeta$ direction can now be obtained by the integral of Equation (27) over the waveguide cross section. Thus, the output power transmission is given by

$$
T=\frac{1}{2} \int_{0}^{2 \pi} \int_{0}^{a} \operatorname{Re}\left\{E_{r} H_{\theta}{ }^{*}-E_{\theta} H_{r}{ }^{*}\right\} r d r d \theta \quad .
$$

In a linear lossy medium, the solution is obtained by replacing the permitivity $\epsilon$ by $\epsilon_{c}=\epsilon-j(\sigma / \omega)$ in the preceding mathematical expressions, where $\epsilon_{c}$ is the complex dielectric constant and $\sigma$ is the conductivity of the medium. The complex Bessel functions are computed by using NAG subroutine (The Numerical Algorithms Group (NAG)).

Several examples computed on a Unix system are presented in the next sections, in order to demonstrate the results of this proposed method for the helical waveguide, based on one bending and two bendings, for practical cases. 


\section{Numerical Results}

This section presents several examples that demonstrate features of the proposed mode model derived in the previous section. The cross-section of the curved waveguide (Figure 1(d)) is made of a tube of various types of material, a metallic layer, and a dielectric layer upon it. The next examples represent the case of the hollow waveguide with a metallic layer $(\mathrm{Ag})$ coated by a thin dielectric layer (AgI). For silver having a conductivity of $6.14 \times 10^{7}(\mathrm{ohm} \cdot \mathrm{m})^{-1}$ and the skin depth at $10.6 \mu \mathrm{m}$ is $1.207 \times 10^{-8} \mathrm{~m}$.

The results of the output transverse components of the fields and the output power density $\left(\left|S_{a v}\right|\right)$ (e.g., Figure 5(a)) show the behavior of the solutions for the TEM $M_{00}$ mode in excitation, for the straight waveguide $(R \rightarrow \infty)$. The result of the output power density (Figure 5(a)) is compared also to the result of published experimental data (Croitoru N. et al., 1997) as shown also in Fig. 5(b)). This comparison shows good agreement (a Gaussian shape) as expected, except for the secondary small propagation mode. The experimental result (Figure 5(b)) is affected by the additional parameters (e.g., the roughnes of the internal wall of the waveguide) which are not taken theoretically into account.

In this example, the length of the straight waveguide is $1 \mathrm{~m}$, the diameter (2a) of the waveguide is $2 \mathrm{~mm}$, the thickness of the dielectric layer $\left[d_{(\mathrm{AgI})}\right]$ is $0.75 \mu \mathrm{m}$, and the minimum spot-size $\left(w_{0}\right)$ is $0.3 \mathrm{~mm}$. The refractive indices of the air, the dielectric layer $(\mathrm{AgI})$ and the metallic layer $(\mathrm{Ag})$ are $n_{(0)}=1, n_{(\mathrm{AgI})}=2.2$, and $n_{(\mathrm{Ag})}=13.5-j 75.3$, respectively. The value of the refractive index of the material at a wavelength of $\lambda=10.6 \mu \mathrm{m}$ is taken from the table compiled by Miyagi, et al. (Miyagi et al., 1984).

The toroidal dielectric waveguide is demonstrated in Figure 5(c). The experimental result that was received in the laboratory of Croitoru at Tel-Aviv University is demonstrated in Figure 5(d). This experimental result was obtained from the measurements of the transmitted $\mathrm{CO}_{2}$ laser radiation $(\lambda=10.6 \mu \mathrm{m})$ propagation through a hollow tube covered on the bore wall with silver and silver-iodide layers (Figure 1(d)), where the initial diameter (ID) is $1 \mathrm{~mm}$ (namely, small bore size).

The output modal profile is greatly affected by the bending, and the theoretical and experimental results (Figure $5(\mathrm{c})-5(\mathrm{~d})$ ) show that in addition to the main propagation mode, several other secondary modes and asymmetric output shape appear. The amplitude of the output power density $\left(\left|S_{a v}\right|\right)$ is small as the bending radius $(\mathrm{R})$ is small, and the shape is far from a Gaussian shape. This result agrees with the experimental results, but not for all the propagation modes. The experimental result (Figure 5(d)) is affected by the bending and additional parameters (e.g., the roughnes of the internal wall of the waveguide) which are not taken theoretically into account. In this example, a $=0.5 \mathrm{~mm}, \mathrm{R}=0.7 \mathrm{~m}, \phi=\pi / 2$, and $\zeta=1 \mathrm{~m}$. The thickness of the dielectric layer $\left[d_{(A g I)}\right]$ is $0.75 \mu \mathrm{m}$ (Figure 1(d)), and the minimum spot size $\left(w_{0}\right)$ is $0.2 \mathrm{~mm}$. The values of the refractive indices of the air, the dielectric layer $(\mathrm{AgI})$ and the metallic layer $(\mathrm{Ag})$ are $n_{(0)}=1, n_{(\mathrm{AgI})}=2.2$, and $n_{(\mathrm{Ag})}=13.5-j 75.3$, respectively. In both theoretical and experimental results (Figures 5(c)-5(d)) the shapes of the output power density for the curved waveguide are not symmetric.

The toroidal waveguide is demonstrated in Figure 6. The theoretical mode-model's result and the experimental result (Morhaim O. et al., 1991) are demonstrated in normalized units where the length of the curved waveguide $(\zeta)$ is $0.55 \mathrm{~m}$, the diameter (2a) of the waveguide is $2.4 \mathrm{~mm}$, and the minimum spot size $\left(w_{0}\right)$ is $0.1 \mathrm{~mm}$. This comparison (Figure 6) between the theoretical mode-model and the experimental data (Morhaim O. et al., 1991) shows an approximated result. For all the examples, our theoretical mode-model takes into account only the dielectric losses and the bending losses, in conjunction with the problem of the propagation through a curved waveguide. The experimental result (Morhaim O. et al., 1991) takes into account additional parameters (e.g., the roughnes of the internal wall of the waveguide) which are not taken theoretically into account. For small values of the bending $(1 / R)$ in the case of small step's angle, the output power transmission is large and decreases with increasing the bending.

The contribution of this paper is demonstrated in Figures 7, 8, 9(a)-9(d) and in Figures 10(a)-10(b), in order to understand the influence of the step's angle $\left(\delta_{p}\right)$ and the radius of the cylinder $(\mathrm{R})$ on the output power transmission, as defined in Eq. (28). Likewise, in order to improve the output power transmission of all section of the CurvedStraight waveguide (Figure 2(a)), of the Straight-Curved waveguide (Figure 2(b)), or the Curved-Straight-Curved waveguide (Figure 3), we have to find the relevant parameters that are needed, based on the output power transmission of the first helical waveguide. Figure 7 represents the effect of the radius of the cylinder on the output power transmission for the first section of the helical waveguide (Figure 1(a)), for $\delta_{p}=0$, where $\delta_{p}$ is the step's angle of the helix. The output power transmission as a function of the radius of the cylinder $(\mathrm{R})$ of the helix is 
shown in Figure 7 where the length of the first section of the helical waveguide $\left(\zeta_{1}\right)$ is $1 \mathrm{~m}$, and where the values of the spot-size $\left(w_{0}\right)$ are $0.06 \mathrm{~mm}$ and $0.15 \mathrm{~mm}$. The diameter (2a) of the waveguide is $2 \mathrm{~mm}$, and the value of the refractive index of the material at a wavelength of $\lambda=10.6 \mu \mathrm{m}$. The output power transmission (T) is at least equal to $75 \%(75 \% \leq \mathrm{T} \leq 100 \%)$. The optimum result is obtained where the spot-size $\left(w_{0}\right)$ is $0.06 \mathrm{~mm}$.

Figure 8 demonstrates the influence of the step's angle and the radius of the cylinder $(\mathrm{R})$ on the output power transmission. Figure 8 is demonstrated for six values of $\delta_{p}\left(\delta_{p}=0.0,0.2,0.4,0.6,0.8,1.0\right)$, where the length of the first section of the helical waveguide $\left(\zeta_{1}\right)$ is $1 \mathrm{~m}$, and where the values of the spot-size $\left(w_{0}\right)$ is $0.06 \mathrm{~mm}$. The diameter (2a) of the waveguide is $2 \mathrm{~mm}$, and the value of the refractive index of the material at a wavelength of $\lambda=10.6 \mu \mathrm{m}$. The output power transmission (T) is at least equal to $75 \%(75 \% \leq \mathrm{T} \leq 100 \%)$. For an arbitrary value of $\mathrm{R}$, the output power transmission is large for large values of $\delta_{p}$ and decreases with decreasing the value of $\delta_{p}$. On the other hand, for an arbitrary value of $\delta_{p}$, the output power transmission is large for large values of $\mathrm{R}$ and decreases with decreasing the value of R. Note that for small values of the step's angle, the radius of the curvature of the helix can be approximated by the radius of the cylinder $(\mathrm{R})$. In this case, the output power transmission is large for small values of the bending $(1 / R)$, and decreases with increasing the bending. Thus, this model can be a useful tool to find the parameters $\left(\delta_{p}\right.$ and $\left.\mathrm{R}\right)$ which will give us the improved results (output power transmission) of a helical hollow waveguide in the cases of space curved waveguides. Figures 9(a)-9(d) show the results of the output transverse components of the field where $\delta_{p}=0.5, R_{1}=0.5 \mathrm{~m}, R_{2}=0.4 \mathrm{~m}, \phi_{1}=\pi, \phi_{2}=\pi / 2$, $\mathrm{a}=1 \mathrm{~mm}, d_{(A g I)}=0.75 \mu \mathrm{m}, \lambda=10.6 \mu \mathrm{m}, w_{0}=0.06 \mathrm{~mm}, n_{(0)}=1, n_{(A g I)}=2.2$, and $n_{(A g)}=13.5-\mathrm{j} 75.3$. The output transverse components of the field show that in addition to the main propagation mode, several other secondary modes appear.

Figures 10(a)-10(b) show the results of the output power density in two cases of the helical waveguide with two bendings, for $\delta_{p}=0.5$ and for $\delta_{p}=1$, where $R_{1}=0.5 \mathrm{~m}, R_{2}=0.4 \mathrm{~m}, \phi_{1}=\pi, \phi_{2}=\pi / 2, \mathrm{a}=1 \mathrm{~mm}, d_{(A g I)}=0.75 \mu \mathrm{m}$, $\lambda=10.6 \mu \mathrm{m}, w_{0}=0.06 \mathrm{~mm}, n_{(0)}=1, n_{(A g I)}=2.2$, and $n_{(A g)}=13.5-\mathrm{j} 75.3$. By increasing only the parameter of the step's angle of the helical waveguide with two bendings $\left(R_{1}=0.5 \mathrm{~m}, R_{2}=0.4 \mathrm{~m}\right)$ from $\delta_{p}=0.5$ to $\delta_{p}=1$, the results of the output power density of the field are changed, and the amplitude of the output power density in Figure (10a) is smaller as regart to the amplitude of the output power density in Figure (10b). The amplitude is small as the step's angle of the helical waveguide is small.

The output results are greatly affected by the step's angle $\left(\delta_{p}\right)$, by the radius of the cylinder $(\mathrm{R})$ of the helix, and by the spot size $\left(w_{0}\right)$. We can solve practical problems in the case of the flexible hollow waveguide, based on one bending and two bendings. Let us assume that the helical waveguide consists of two different bendings as shown in Figures 2(a) and 2(b), where one of the sections is a bending helical waveguide, and the other section is a straight waveguide. The Curved-Straight waveguide is shown in Figure 2(a) and the Straight-Curved waveguide is shown in Figure 2(b). The helical waveguide that consists of three bendings (Curved-Straight-Curved waveguide) is shown in Figure 3.

Let us assume that the requirement in these examples of the waveguides with two or three sections is that the output power transmission ( $\mathrm{T})$ is at least equal to $80 \%(80 \% \leq \mathrm{T} \leq 100 \%)$. The practical results of the output power transmission are greatly affected by the step's angle $\left(\delta_{p}\right)$, by the radius of the cylinder $(\mathrm{R})$ of the helix, and by the spot size $\left(w_{0}\right)$. Thus, in order to improve the output power transmission of the Curved-Straight waveguide (Figure 2(a)), of the Straight-Curved waveguide (Figure 2(b)), or the Curved-Straight-Curved waveguide (Figure $3)$, the relevant parameters of the step's angle $\left(\delta_{p}=0.6,0.8,1.0\right)$, the radius of the cylinder $(\mathrm{R}>1)$, and the spot size $\left(w_{0}=0.06 \mathrm{~mm}\right)$ are needed, according to Figure 8 . In the cases of $\mathbf{R} \leq 1$, the step's angle $\left(\delta_{p}=1.0\right)$ and the spot size $\left(w_{0}=0.06 \mathrm{~mm}\right)$ are needed to improve the output power transmission.

This mode model can be a useful tool in order to determine the optimal conditions for practical applications (output fields, output power density and output power transmission as function of the step's angle and the radius of the cylinder of the helix). This mode model can be a useful tool to improve the output results in all the cases of the hollow waveguides, based on one bending and two bendings, for industrial and medical regimes.

\section{Conclusions}

This paper presents an improved approach for the propagation of EM fields along a helical hollow waveguide, based on one bending and two bendings. The objective is to develop a mode model for infrared (IR) wave propagation, in order to represent the effect of the radius of the cylinder of the helix and the step's angle on the output fields and the output power transmission. This model enables us to understand more precisely the influence of the step's angle and the radius of the cylinder of the helix on the output results of each section (bending). The 
examples represent the case of the hollow waveguide with a metallic layer $(\mathrm{Ag})$ coated by a thin dielectric layer (AgI).

The results of the output transverse components of the fields and the output power density $\left(\left|S_{a v}\right|\right)$ (e.g., Figure 5(a)) show the behavior of the solutions for the $T E M_{00}$ mode in excitation, for the straight waveguide $(R \rightarrow \infty)$. The result of the output power density (Figure 5(a)) was compared also to the result of published experimental data (Croitoru N. et al., 1997) as shown also in Figure 5(b). This comparison shows good agreement (a Gaussian shape) as expected, except for the secondary small propagation mode. The experimental result (Figure 5(b)) is affected by the additional parameters (e.g., the roughnes of the internal wall of the waveguide) which are not taken theoretically into account.

The toroidal dielectric waveguide was demonstrated in Figure 5(c). Figure 5(d) shows the experimental result that was received in the laboratory of Croitoru at Tel-Aviv University. This experimental result was obtained from the measurements of the transmitted $\mathrm{CO}_{2}$ laser radiation $(\lambda=10.6 \mu \mathrm{m})$ propagation through a hollow tube covered on the bore wall with silver and silver-iodide layers (Figure 1(d)), where the initial diameter (ID) is $1 \mathrm{~mm}$ (namely, small bore size). The output modal profile is greatly affected by the bending, and the theoretical and experimental results (Figures 5(c)-5(d)) show that in addition to the main propagation mode, several other secondary modes and asymmetric output shape appear. The amplitude of the output power density $\left(\left|S_{a v}\right|\right)$ is small as the bending radius ( $\mathrm{R}$ ) is small, and the shape is far from a Gaussian shape. This result agrees with the experimental results, but not for all the propagation modes. The experimental result (Figure 5(d)) is affected by the bending and additional parameters (e.g., the roughnes of the internal wall of the waveguide) which are not taken theoretically into account. In both theoretical and experimental results (Figures 5(c)-5(d)) the shapes of the output power density for the curved waveguide are not symmetric.

The comparison (Figure 6) between the theoretical mode-model and the experimental data (Morhaim O. et al., 1991) shows an approximated result. For all the examples, our theoretical mode-model takes into account only the dielectric losses and the bending losses, in conjunction with the problem of the propagation through a curved waveguide. The experimental result (Morhaim O. et al., 1991) takes into account additional parameters (e.g., the roughnes of the internal wall of the waveguide) which are not taken theoretically into account. For small values of the bending $(1 / \mathrm{R})$ in the case of small step's angle, the output power transmission is large and decreases with increasing the bending.

The contribution of this paper was demonstrated in Figures 7, 8, 9(a)-9(d) and in Figures 10(a)-10(b), in order to understand the influence of the step's angle $\left(\delta_{p}\right)$ and the radius of the cylinder $(\mathrm{R})$ on the output power transmission, as defined in Eq. (28). Likewise, in order to improve the output power transmission of all section of the Curved-Straight waveguide (Figure 2(a)), of the Straight-Curved waveguide (Figure 2(b)), or the CurvedStraight-Curved waveguide (Figure 3), we have to find the relevant parameters that are needed, based on the output power transmission of the first helical waveguide.

Figure 7 represents the effect of the radius of the cylinder on the output power transmission for the first section of the helical waveguide (Figure 1(a)), for $\delta_{p}=0$, where $\delta_{p}$ is the step's angle of the helix. The output power transmission as a function of the radius of the cylinder (R) of the helix is shown in Figure 7 where the length of the first section of the helical waveguide $\left(\zeta_{1}\right)$ is $1 \mathrm{~m}$, and where the values of the spot-size $\left(w_{0}\right)$ are $0.06 \mathrm{~mm}$ and $0.15 \mathrm{~mm}$. The diameter (2a) of the waveguide is $2 \mathrm{~mm}$, and the value of the refractive index of the material at a wavelength of $\lambda=10.6 \mu \mathrm{m}$. The output power transmission (T) is at least equal to $75 \%$ (75\% $\leq \mathrm{T} \leq 100 \%)$. The optimum result is obtained where the spot-size $\left(w_{0}\right)$ is $0.06 \mathrm{~mm}$.

Figure 8 demonstrates the influence of the step's angle and the radius of the cylinder $(\mathrm{R})$ on the output power transmission. Figure 8 is demonstrated for six values of $\delta_{p}\left(\delta_{p}=0.0,0.2,0.4,0.6,0.8,1.0\right)$, where the length of the first section of the helical waveguide $\left(\zeta_{1}\right)$ is $1 \mathrm{~m}$, and where the values of the spot-size $\left(w_{0}\right)$ is $0.06 \mathrm{~mm}$. The diameter (2a) of the waveguide is $2 \mathrm{~mm}$, and the value of the refractive index of the material at a wavelength of $\lambda=10.6 \mu \mathrm{m}$. The output power transmission (T) is at least equal to $75 \%(75 \% \leq \mathrm{T} \leq 100 \%)$.

For an arbitrary value of $\mathrm{R}$, the output power transmission is large for large values of $\delta_{p}$ and decreases with decreasing the value of $\delta_{p}$. On the other hand, for an arbitrary value of $\delta_{p}$, the output power transmission is large for large values of R and decreases with decreasing the value of R. Note that for small values of the step's angle, the radius of the curvature of the helix can be approximated by the radius of the cylinder (R). In this case, the output power transmission is large for small values of the bending $(1 / R)$, and decreases with increasing the bending. Thus, this model can be a useful tool to find the parameters ( $\delta_{p}$ and $\left.\mathrm{R}\right)$ which will give us the improved 
results (output power transmission) of a helical hollow waveguide in the cases of space curved waveguides.

The output transverse components of the field (Figures 9(a)-9(d)) show that in addition to the main propagation mode, several other secondary modes appear. Figures 10(a)-10(b) show the results of the output power density in two cases of the helical waveguide with two bendings, for $\delta_{p}=0.5$ and for $\delta_{p}=1$, where $R_{1}=0.5 \mathrm{~m}, R_{2}=$ $0.4 \mathrm{~m}, \phi_{1}=\pi, \phi_{2}=\pi / 2, \mathrm{a}=1 \mathrm{~mm}, d_{(A g I)}=0.75 \mu \mathrm{m}, \lambda=10.6 \mu \mathrm{m}, w_{0}=0.06 \mathrm{~mm}, n_{(0)}=1, n_{(\mathrm{AgI})}=2.2$, and $n_{(A g)}=13.5-\mathrm{j} 75.3$. By increasing only the parameter of the step's angle of the helical waveguide with two bendings $\left(R_{1}=0.5 \mathrm{~m}, R_{2}=0.4 \mathrm{~m}\right)$ from $\delta_{p}=0.5$ to $\delta_{p}=1$, the results of the output power density of the field are changed, and the amplitude of the output power density in Figure (10a) is smaller as regart to the amplitude of the output power density in Figure (10b). The amplitude is small as the step's angle of the helical waveguide is small.

We can solve practical problems in the case of the flexible hollow waveguide, based on one bending and two bendings. The practical results of the output power transmission are greatly affected by the step's angle $\left(\delta_{p}\right)$, by the radius of the cylinder $(\mathrm{R})$ of the helix, and by the spot size $\left(w_{0}\right)$. Thus, in order to improve the output power transmission of the Curved-Straight waveguide (Figure 2(a)), of the Straight-Curved waveguide (Figure 2(b)), or of the Curved-Straight-Curved waveguide (Figure 3), the relevant parameters of the step's angle $\left(\delta_{p}=0.6,0.8\right.$, $1.0)$, the radius of the cylinder $(\mathrm{R}>1)$, and the spot size $\left(w_{0}=0.06 \mathrm{~mm}\right)$ are needed, according to Figure 8 . In the cases of $\mathrm{R} \leq 1$, the step's angle $\left(\delta_{p}=1.0\right)$ and the spot size $\left(w_{0}=0.06 \mathrm{~mm}\right)$ are needed to improve the output power transmission.

This mode model can be a useful tool in order to determine the optimal conditions for practical applications (output fields, output power density and output power transmission as function of the step's angle and the radius of the cylinder of the helix). This mode model can be a useful tool to improve the output results in all the cases of the hollow waveguides, based on one bending and two bendings, for industrial and medical regimes.

\section{References}

Baden Fuller, A. J. (1969). Microwaves, Pergamon Press, A. Wheaton and Co. Ltd, Oxford, Chap. 5, $118-120$.

Bienstman, P., Roelens, M., Vanwolleghem, M., \& Baets, R. (2002). Calculation of bending losses in dielectric waveguides using eigenmode expansion and perfectly matched layers. IEEE Photon. Technol. Lett., 14, 164-166.

Croitoru, N., Goldenberg, E., Mendlovic, D., Ruschin, S., \& Shamir, N. (1986). Infrared chalcogenide tube waveguides. SPIE, 618, 140-145.

Croitoru, N., Inberg, A., Oksman, M., \& Ben-David M. (1997). Hollow silica, metal and plastic waveguides for hard tissue medical applications. SPIE, 2977, 30-35.

Harrington, J. A., \& Matsuura, Y. (1995). Review of hollow waveguide technology. SPIE, 2396.

Harrington, J. A., Harris, D. M., \& Katzir, A. (1995). Biomedical Optoelectronic Instrumentation, 4-14.

Harrington, J. A. (2000). A review of IR transmitting, hollow waveguides. Fiber and Integrated Optics, 19, 211-228.

Jahnke, E., \& Emde, F. (1945). Tables of Functions with Formulae and Curves. Dover Publications, New York, Chap. 8, 166.

Kark, K. W. (1991). Perturbation analysis of electromagnetic eigenmodes in toroidal waveguides. IEEE Trans. Microwave Theory Tech., 39, 631-637.

Lewin, L., Chang, D. C., \& Kuester, E. F. (1977). Electromagnetic Waves and Curved Structures. Peter Peregrinus Ltd., London, Chap. 6, 58-68.

Marcatili, E. A. J., \& Schmeltzer, R. A. (1964). Hollow metallic and dielectric waveguides for long distance optical transmission and lasers. Bell Syst. Tech. J., 43, 1783-1809.

Marhic, M. E. (1981). Mode-coupling analysis of bending losses in IR metallic waveguides. Appl. Opt., 20, 3436-3441.

Melloni, A., Carniel, F., Costa, R., \& Martinelli, M. (2001). Determination of bend mode characteristics in dielectric waveguides. J. Lightwave Technol., 19, 571-577. 
Menachem, Z., Croitoru, N., \& Aboudi, J. (2002). Improved mode model for infrared wave propagation in a toroidal dielectric waveguide and applications. Opt. Eng., 41, 2169-2180.

Menachem, Z. (2003). Wave propagation in a curved waveguide with arbitrary dielectric transverse profiles. Journal of Electromagnetic Waves and Applications, 17(10), 1423-1424, and Progress In Electromagnetics Research, PIER, 42, 173-192.

Menachem, Z., \& Mond, M. (2006). Infrared wave propagation in a helical waveguide with inhomogeneous cross section and applications. Progress In Electromagnetics Research, PIER, 61, 159-192.

Menachem, Z., \& Haridim, M. (2009). Propagation in a helical waveguide with inhomogeneous dielectric profiles in rectangular cross section. Progress In Electromagnetics Research B, 16, 155-188.

Menachem, Z. (2010). Flexible hollow waveguide with two bendings for small values of step angles, and applications. Progress In Electromagnetics Research B, 21, 347-383.

Menachem, Z., \& Tapuchi, S. (2010). Helical waveguide with two bendings, and applications. Progress In Electromagnetics Research B, 26, 115-147.

Mendlovic, D., Goldenberg, E., Ruschin, S., Dror J., \& Croitoru, N. (1989). Ray model for transmission of metallic-dielectric hollow bent cylindrical waveguides. Appl. Opt., 28, 708-712.

Miyagi, M., Harada, K., \& Kawakami, S. (1984). Wave propagation and attenuation in the general class of circular hollow waveguides with uniform curvature. IEEE Trans. Microwave Theory Tech., 32, 513-521.

Morhaim, O., Mendlovic, D., Gannot, I., Dror, J., \& Croitoru, N. (1991). Ray model for transmission of infrared radiation through multibent cylindrical waveguides. Opt. Eng., 30, 1886-1891.

Olver, F. W. J. (1960). Royal Society Mathematical Tables. Zeros and Associated Values. University Press Cambridge.

The Numerical Algorithms Group (NAG) Ltd. Wilkinson House, Oxford, U.K.

Yariv, A. (1985). Optical Electronics (3rd ed.). Holt-Saunders Int. Editions.

\section{Appendix A.}

The elements $\left(\alpha_{n}{ }^{(1)}, \beta_{n}{ }^{(1)}\right.$, etc), on the left side of (16a) for $\mathrm{n}=1$ are given for an arbitrary value of the step's angle $\left(\delta_{p}\right)$ for the first section of the helical waveguide by:

$$
\begin{gathered}
\alpha_{1}{ }^{(1) m m^{\prime}}=\pi\left(s^{2}+\beta_{1 m^{\prime}}^{2}\right)\left[\left(s^{2}+k_{0}^{2}\right) G_{00}^{(1) m m^{\prime}}+k_{0}^{2} G_{01}^{(1) m m^{\prime}}\right] \\
+\pi \frac{1}{R_{1}^{4}} k_{0}^{2} s^{2}\left(\frac{1}{4} \cos ^{4}\left(\delta_{p}\right) G_{02}^{(1) m m^{\prime}}+\frac{1}{2} \cos ^{4}\left(\delta_{p}\right) G_{03}^{(1) m m^{\prime}}\right) \\
+\pi k_{0}^{2}\left\{s^{2} G_{01}^{(1) m m^{\prime}}+G_{05}^{(1) m m^{\prime}}+\frac{1}{R_{1}^{2}}\left(G_{00}^{(1) m m^{\prime}}+G_{01}^{(1) m m^{\prime}}\right)+\frac{3}{2 R_{1}^{2}} \beta_{1 m^{\prime}}^{2} \cos ^{4}\left(\delta_{p}\right)\left(G_{02}^{(1) m m^{\prime}}+G_{03}^{(1) m m^{\prime}}\right)\right. \\
\left.+\frac{1}{4 R_{1}^{4}} \cos ^{4}\left(\delta_{p}\right)\left(G_{02}^{(1) m m^{\prime}}+G_{03}^{(1) m m^{\prime}}\right)+\frac{1}{8 R_{1}^{4}} \cos ^{8}\left(\delta_{p}\right)\left(G_{06}^{(1) m m^{\prime}}+G_{07}^{(1) m m^{\prime}}\right)\right\} \\
+\pi s^{2}\left[G_{08}^{(1) m m^{\prime}}+\frac{1}{2 R_{1}^{2}} \cos ^{2}\left(\delta_{p}\right) G_{00}^{(1) m m^{\prime}}+\frac{1}{4 R_{1}^{2}}\left(\cos ^{4}\left(\delta_{p}\right) \beta_{1 m^{\prime}}^{2} G_{02}^{(1) m m^{\prime}}+\cos ^{2}\left(\delta_{p}\right) G_{09}^{(1) m m^{\prime}}\right)\right. \\
+\frac{1}{2 R_{1}^{2}} \frac{\left.P_{1 m^{\prime}} \cos ^{2}\left(\delta_{p}\right)\left(G_{10}^{(1) m m^{\prime}}+\frac{1}{2} \cos ^{2}\left(\delta_{p}\right) G_{11}^{(1) m m^{\prime}}\right)\right]}{\beta_{1}^{(1) m m^{\prime}}=-j \omega \mu_{0} \pi s\left\{G_{13}^{(1) m m^{\prime}}+\left(\frac{1}{2} \cos ^{2}\left(\delta_{p}\right)+\frac{3}{4} \cos ^{4}\left(\delta_{p}\right)\right) \frac{1}{R_{1}^{2}} G_{14}^{(1) m m^{\prime}}+\left(\frac{1}{2}+\cos ^{2}\left(\delta_{p}\right)\right) \frac{1}{R_{1}^{2}} G_{15}^{(1) m m^{\prime}}\right.} \\
+\pi k_{0}^{4} \cos ^{4}\left(\delta_{p}\right)\left[\frac{3}{2 R_{1}^{2}}\left(G_{03}^{(1) m m^{\prime}}+G_{04}^{(1) m m^{\prime}}\right)+\frac{1}{8 R_{1}^{4}} \cos ^{8}\left(\delta_{p}\right)\left(G_{07}^{(1) m m^{\prime}}+G_{12}^{(1) m m^{\prime}}\right)\right],
\end{gathered}
$$




$$
\left.-\frac{1}{2 R_{1}^{2}} G_{00}^{(1) m m^{\prime}}-\cos ^{2}\left(\delta_{p}\right) \frac{1}{R_{1}^{2}} \frac{P_{1 m^{\prime}}^{\prime}}{a} G_{16}^{(1) m m^{\prime}}\right\},
$$

The elements of the matrices $\left(G_{00}^{(1) m m^{\prime}}\right.$, etc.) are given by:

$$
\begin{gathered}
G_{00}^{(1) m m^{\prime}}=\int_{0}^{a} J_{1}\left(P_{1 m^{\prime}} \frac{r}{a}\right) J_{1}\left(P_{1 m} \frac{r}{a}\right) r d r \delta_{1 n}, \quad G_{01}^{(1) m m^{\prime}}=\int_{0}^{a} g(r) J_{1}\left(P_{1 m^{\prime}} \frac{r}{a}\right) J_{1}\left(P_{1 m} \frac{r}{a}\right) r d r \delta_{1 n}, \\
G_{02}^{(1) m m^{\prime}}=\int_{0}^{a} J_{1}\left(P_{1 m^{\prime}} \frac{r}{a}\right) J_{1}\left(P_{1 m} \frac{r}{a}\right) r^{3} d r \delta_{1 n}, \quad G_{03}^{(1) m m^{\prime}}=\int_{0}^{a} g(r) J_{1}\left(P_{1 m^{\prime}} \frac{r}{a}\right) J_{1}\left(P_{1 m} \frac{r}{a}\right) r^{3} d r \delta_{1 n}, \\
G_{04}^{(1) m m^{\prime}}=\int_{0}^{a} g^{2}(r) J_{1}\left(P_{1 m^{\prime}} \frac{r}{a}\right) J_{1}\left(P_{1 m} \frac{r}{a}\right) r^{3} d r \delta_{1 n}, \quad G_{05}^{(1) m m^{\prime}}=\int_{0}^{a} k^{2} g(r) J_{1}\left(P_{1 m^{\prime}} \frac{r}{a}\right) J_{1}\left(P_{1 m} \frac{r}{a}\right) r d r \delta_{1 n}, \\
G_{06}^{(1) m m^{\prime}}=\int_{0}^{a} J_{1}\left(P_{1 m^{\prime}} \frac{r}{a}\right) J_{1}\left(P_{1 m} \frac{r}{a}\right) r^{5} d r \delta_{1 n}, \quad G_{07}^{(1) m m^{\prime}}=\int_{0}^{a} g(r) J_{1}\left(P_{1 m^{\prime}} \frac{r}{a}\right) J_{1}\left(P_{1 m} \frac{r}{a}\right) r^{5} d r \delta_{1 n}, \\
G_{08}^{(1) m m^{\prime}}=\int_{0}^{a} g_{r}\left(\frac{P_{1 m^{\prime}}}{a}\right) J_{1}^{\prime}\left(P_{1 m^{\prime}} \frac{r}{a}\right) J_{1}\left(P_{1 m} \frac{r}{a}\right) r d r, \quad G_{09}^{(1) m m^{\prime}}=\int_{0}^{a} g_{r} J_{1}\left(P_{1 m^{\prime}} \frac{r}{a}\right) J_{1}\left(P_{1 m} \frac{r}{a}\right) r^{2} d r \delta_{1 n}, \\
G_{10}^{(1) m m^{\prime}}=\int_{0}^{a} J_{1}^{\prime}\left(\frac{P_{1 m^{\prime}}}{a}\right) J_{1}\left(P_{1 m} \frac{r}{a}\right) r^{2} d r \delta_{1 n}, \quad G_{11}^{(1) m m^{\prime}}=\int_{0}^{a} g_{r} J_{1}^{\prime}\left(\frac{P_{1 m^{\prime}}}{a}\right) J_{1}\left(P_{1 m} \frac{r}{a}\right) r^{3} d r \delta_{1 n}, \\
G_{12}^{(1) m m^{\prime}}=\int_{0}^{a} g^{2}(r) J_{1}\left(P_{1 m^{\prime}} \frac{r}{a}\right) J_{1}\left(P_{1 m} \frac{r}{a}\right) r^{5} d r \delta_{1 n}, \quad G_{13}^{(1) m m^{\prime}}=\int_{0}^{a} g_{r} J_{1}^{\prime}\left(\frac{p_{1 m^{\prime}}^{\prime}}{a}\right) J_{1}\left(P_{1 m} \frac{r}{a}\right) d r \delta_{1 n}, \\
G_{14}^{(1) m m^{\prime}}=\int_{0}^{a} g_{r} J_{1}\left(\frac{p_{1 m^{\prime}}^{\prime}}{a}\right) J_{1}\left(P_{1 m} \frac{r}{a}\right) r^{2} d r \delta_{1 n}, \quad G_{15}^{(1) m m^{\prime}}=\int_{0}^{a} J_{1}\left(P_{1 m^{\prime}} \frac{r}{a}\right) J_{1}\left(P_{1 m} \frac{r}{a}\right) r d r \delta_{1 n}, \\
G_{16}^{(1) m m^{\prime}}=\int_{0}^{a} J_{1}^{\prime}\left(\frac{P_{1 m^{\prime}}^{\prime}}{a}\right) J_{1}\left(P_{1 m} \frac{r}{a}\right) r^{2} d r \delta_{1 n},
\end{gathered}
$$

Similarly, the remaining elements are obtained. The coefficients are obtained directly from the algebraic system of equations (16a)-(16d) and are expressed as functions in s-plane. Similarly, the other coefficients are obtained. 


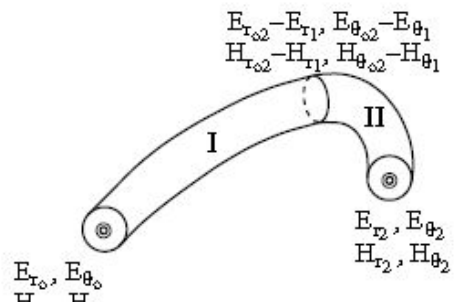

(a)

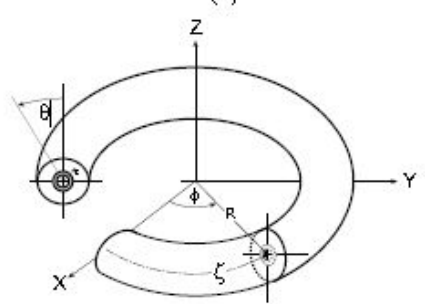

(c)

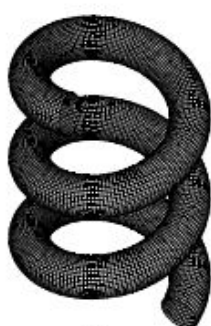

(b)

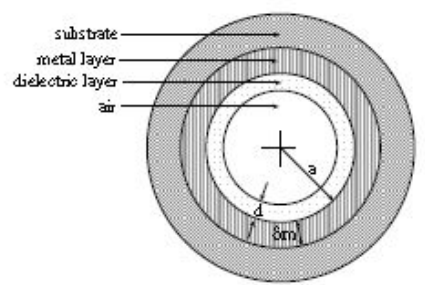

(d)

Figure 1. (a) A general scheme for a helical waveguide with two bendings in the same direction. (b) The helical circular waveguide. (c) A general scheme of the toroidal system $(r, \theta, \zeta)$ and the curved waveguide. (d) A cross-section of the waveguide $(\mathrm{r}, \theta)$

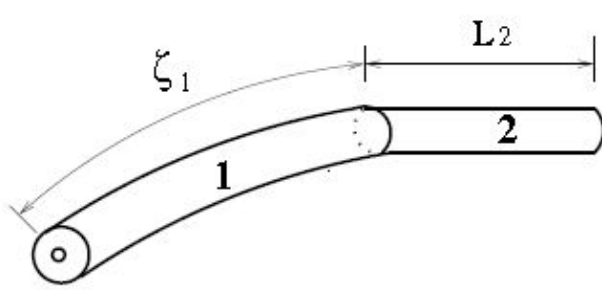

(a)

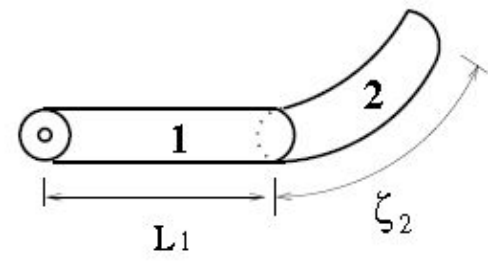

(b)

Figure 2. Practical examples of the flexible hollow waveguide with two sections: (a). Curved-Straight waveguide.

(b). Straight-Curved waveguide

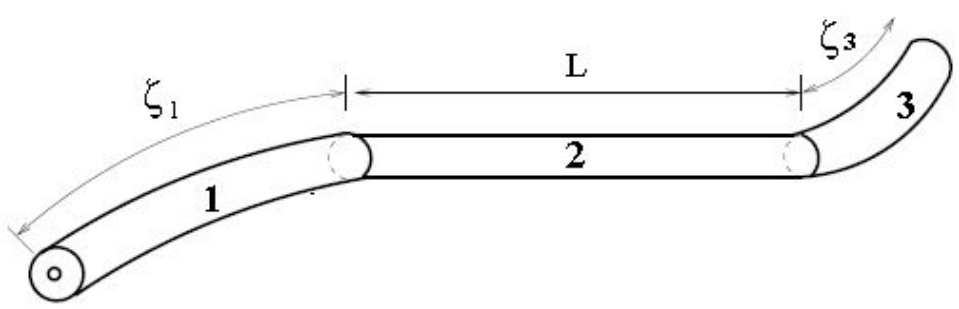

Figure 3. Practical example of the flexible helical waveguide with three sections (Curved-Straight-Curved waveguide) 


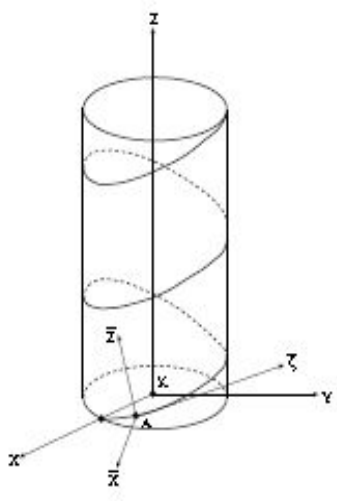

(a)

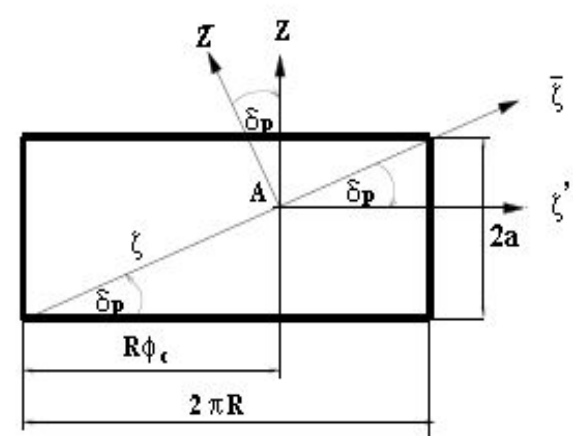

(b)

Figure 4. (a) Rotations and translation of the orthogonal system $(\bar{X}, \bar{\xi}, \bar{Z})$ from point $A$ to the orthogonal system $(X, Y, Z)$ at point $K$. (b) Deployment of the helix
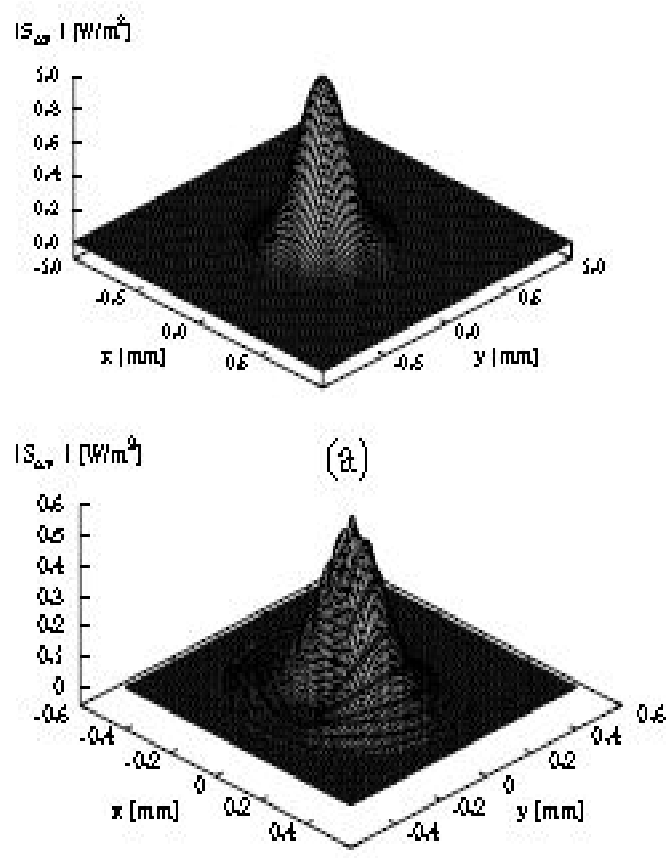

(c)

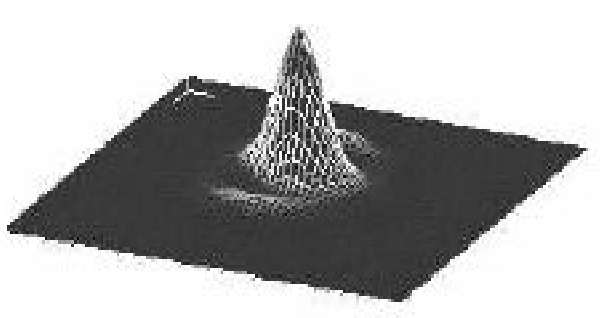

(b)

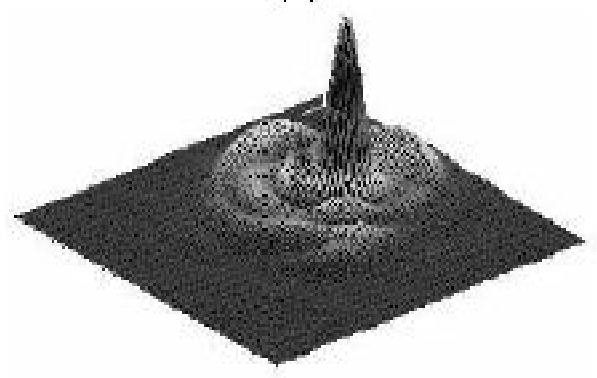

(d)

Figure 5. The output power density for $\mathrm{R} \rightarrow \infty$, where $\mathrm{a}=1 \mathrm{~mm}, w_{0}=0.3 \mathrm{~mm}$, and the length of the straight waveguide is $1 \mathrm{~m}$. (a) theoretical result; (b) experimental result. The output power density for the toroidal dielectric waveguide, where a $=0.5 \mathrm{~mm}, w_{0}=0.2 \mathrm{~mm}, \mathrm{R}=0.7 \mathrm{~m}, \phi=\pi / 2$; and $\zeta=1 \mathrm{~m}$; (c) theoretical result; (d)

experimental result. The other parameters are: $d\left({ }_{A g I}\right)=0.75 \mu \mathrm{m}, \lambda=10.6 \mu \mathrm{m}, ; n_{(0)}=1, n_{(A g I)}=2.2$, and

$$
n_{(A g)}=13.5-j 75
$$




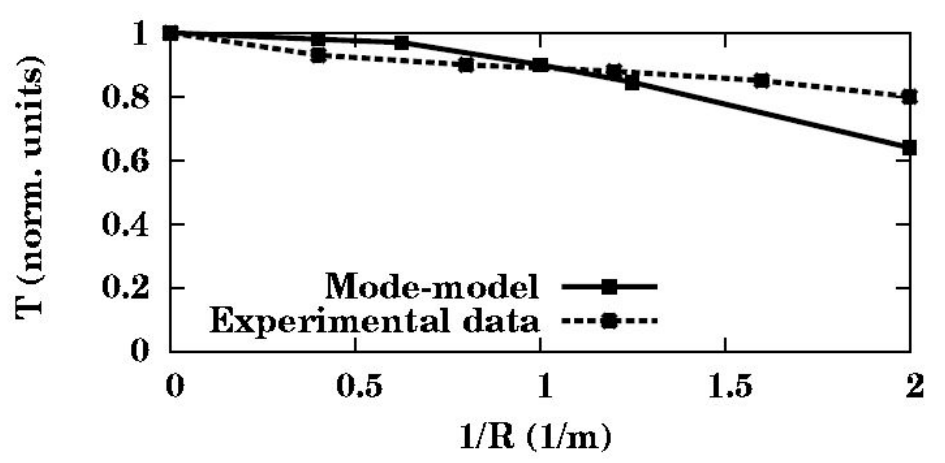

Figure 6. The theoretical mode-model's result and the experimental result (Morhaim et al., 1991) where the hollow metallic waveguide $(\mathrm{Ag})$ is covered inside the walls with a $\mathrm{AgI}$ film. The output power transmission as a function of $1 / \mathrm{R}$ for $\delta_{p}=0$, where $\zeta=0.55 \mathrm{~m}, \mathrm{a}=1.2 \mathrm{~mm}, d(A g I)=0.75 \mu \mathrm{m}, w 0=0.1 \mathrm{~mm}, \lambda=10.6 \mu \mathrm{m}, n(0)=1$, $n(A g I)=2.2$, and $n(A g)=10$

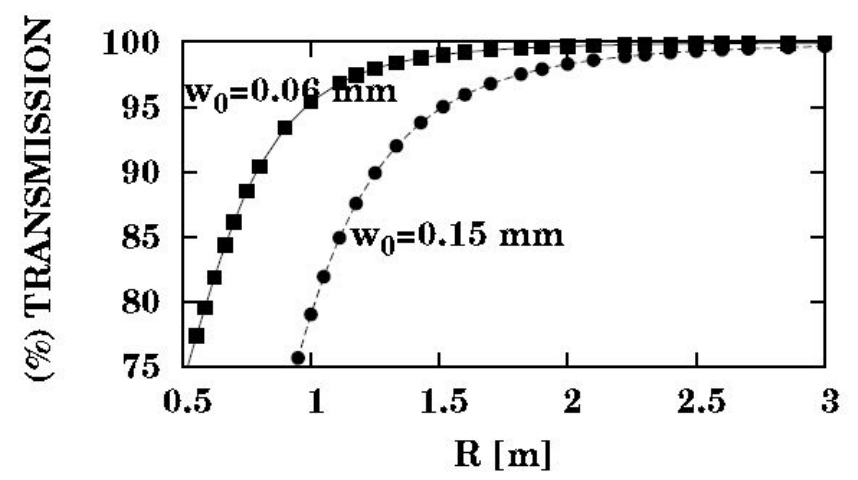

Figure 7. The output power transmission as a function of the radius of the cylinder (R) for $\zeta_{1}=1 \mathrm{~m}$ in two cases of the spot size: (a). $w 0=0.06 \mathrm{~mm}$ (b). $w 0=0.15 \mathrm{~mm}$, for $\delta_{p}=0$. The optimum result is obtained by the first solution of the first section of the helical waveguide

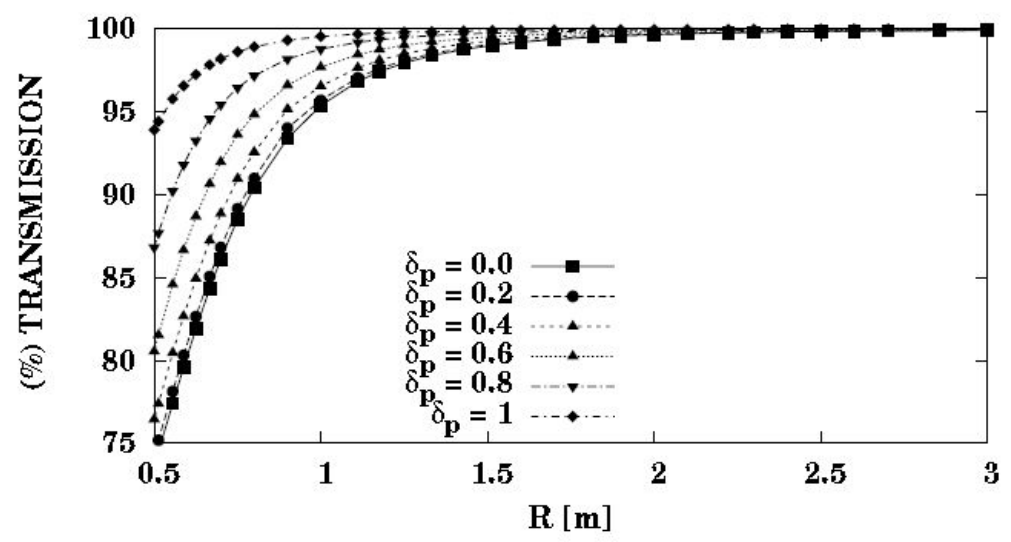

Figure 8. The output power transmission of the helical waveguide as a function of R. Six results are demonstrated for six values of $\delta_{p}\left(\delta_{p}=0.0,0.2,0.4,0.6,0.8,1.0\right)$, where $\zeta_{1}=1 \mathrm{~m}, \mathrm{a}=1 \mathrm{~mm}, w 0=0.06 \mathrm{~mm}$, $n_{d}=2.2$, and $n(A g)=13.5-j 75.3$ 


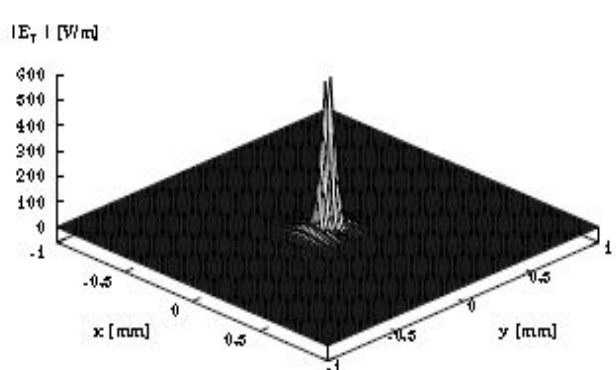

(a)

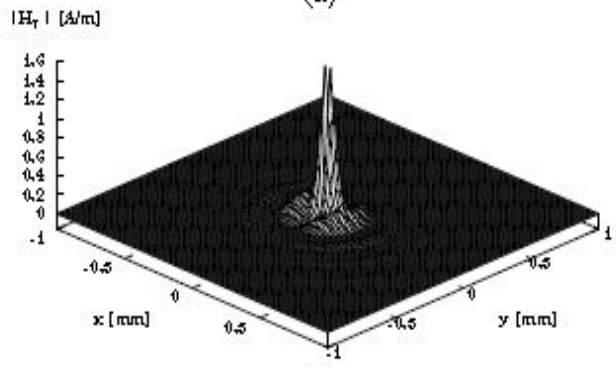

(c)

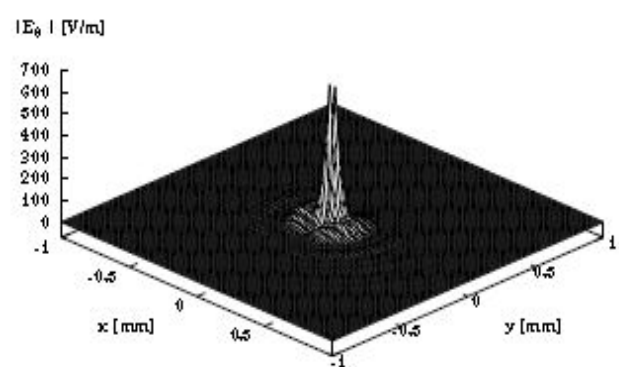

(b)

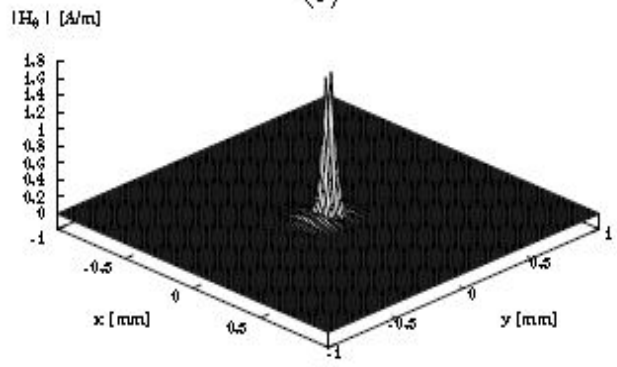

(d)

Figure 9. The solution of the output transverse components (a-d) in the case of the helical waveguide with two bendings, where $\delta p=0.5, R_{1}=0.5 \mathrm{~m}, R_{2}=0.4 \mathrm{~m}, \phi_{1}=\pi ; \phi_{2}=\pi / 2, \mathrm{a}=1 \mathrm{~mm}, d(A g I)=0.75 \mu \mathrm{m}$ $\lambda=10.6 \mu \mathrm{m}, w_{0}=0.06 \mathrm{~mm}, n_{(0)}=1, n_{(A g I)}=2.2$, and $n_{(A g)}=13.5-j 75.3$

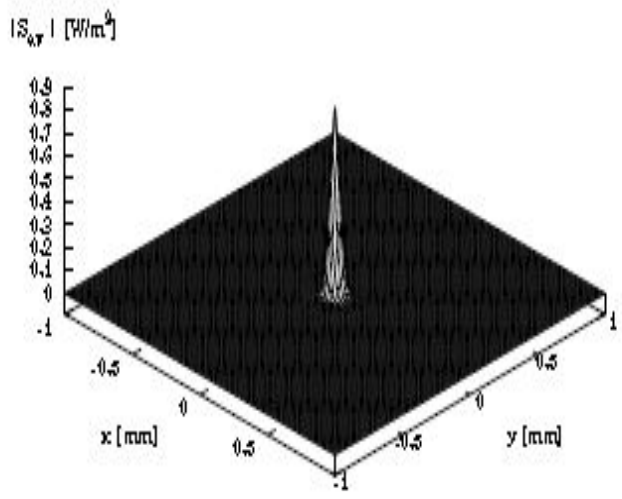

$(\hat{a})$

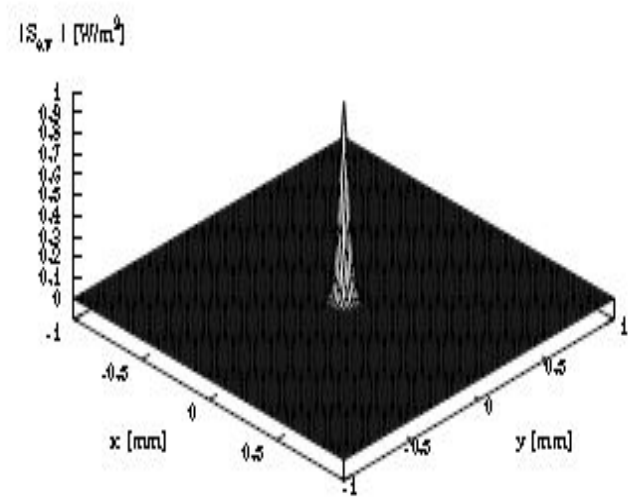

(b)

Figure 10. The solution of the output power density in two cases of the helical waveguide with two bendings, for (a) $\delta p=1, R_{1}=0.5 \mathrm{~m}, R_{2}=0.4 \mathrm{~m}, \phi_{1}=\pi ; \phi_{2}=\pi / 2, \mathrm{a}=1 \mathrm{~mm}, d(A g I)=0.75 \mu \mathrm{m} \lambda=10.6 \mu \mathrm{m}$, $w_{0}=0.06 \mathrm{~mm}, n_{(0)}=1, n_{(A g I)}=2.2$, and $n_{(A g)}=13.5-j 75.3$ 